This item was submitted to Loughborough's Research Repository by the author.

Items in Figshare are protected by copyright, with all rights reserved, unless otherwise indicated.

\title{
Computational investigation into the sensitivity of a simplified vehicle wake to small base geometry changes
}

\section{PLEASE CITE THE PUBLISHED VERSION}

https://doi.org/10.1016/j.jweia.2018.12.010

\section{PUBLISHER}

(C) Elsevier

VERSION

AM (Accepted Manuscript)

\section{PUBLISHER STATEMENT}

This paper was accepted for publication in the journal Journal of Wind Engineering and Industrial Aerodynamics and the definitive published version is available at https://doi.org/10.1016/j.jweia.2018.12.010

LICENCE

CC BY-NC-ND 4.0

\section{REPOSITORY RECORD}

Luckhurst, Sophie, Max Varney, Hao Xia, Martin Passmore, and Adrian P. Gaylard. 2019. "Computational Investigation into the Sensitivity of a Simplified Vehicle Wake to Small Base Geometry Changes". figshare. https://hdl.handle.net/2134/36501. 


\title{
Computational Investigation into the Sensitivity of a Simplified Vehicle Wake to Small Base Geometry Changes
}

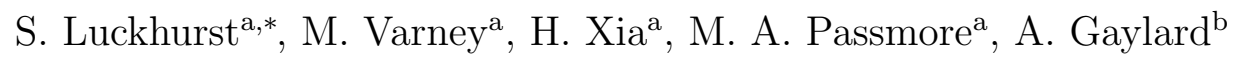 \\ ${ }^{a}$ Department of Aeronautical \& Automotive Engineering, Loughborough University, Loughborough, LE11 \\ 3TU, $U K$ \\ ${ }^{b}$ Jaguar Land Rover, Gaydon, CV35 ORR, UK
}

\begin{abstract}
For vehicles with a squareback geometry, for example Sports Utility Vehicles (SUVs), base pressure drag is a large contributor to overall drag. Simple passive techniques, such as tapering, can reduce drag significantly but at a large aesthetic and functional cost. Therefore, very small base geometry changes have been investigated. An experimentally validated methodology has used Detached Eddy Simulations (DES) to obtain time-averaged and instantaneous data; allowing the effect of horizontal base slats on global forces and wake structures to be presented.

The small geometry modifications have caused substantial changes to the base pressure distribution with the main mechanisms of change being identified and observed close to the model surfaces. A region of separation is seen below each slat corresponding to reduced pressure whilst high pressure regions attributed to stagnation are increased. The combined effect is a statistically significant drag reduction of 4 counts $\left(1\right.$ count $\left.=0.001 \mathrm{C}_{\mathrm{D}}\right)$ when a slat is added at $3 / 4$ of the base height. The results show the scope for very small changes to a simplified road vehicle, in areas that have not previously been explored, to reduce overall drag with minimal aesthetic penalties. This understanding provides the impetus for new approaches in real vehicle development.
\end{abstract}

Keywords: Vehicle Aerodynamics, CFD, Bluff Body, Wake Dynamics

\section{Introduction}

2

$\mathrm{CO}_{2}$ emission targets are becoming increasingly stringent, as demonstrated by Euro3 pean market regulations mandating significantly reduced, manufacturer fleet averages to be

\footnotetext{
*Corresponding author

Email address: s.luckhurst@lboro.ac.uk (S. Luckhurst)
} 
achieved by 2021 [1]. This increases the need for automotive manufacturers to improve vehicle efficiency in order to avoid the financial penalties for failing to meet the required limits. One way to improve efficiency is to reduce the vehicle drag as this means the powertrain has to produce less energy to move or accelerate the vehicle [2]. A drag reduction of just 4 counts is equivalent to a saving of $0.5 \mathrm{gCO}_{2} / \mathrm{km}$ and being over emissions targets by this amount will result in fines of $€ 42.5$ per car sold. Further to this, as we move towards an increased number of electric vehicles, this same drag reduction increases vehicle range by $2 \mathrm{~km}$. This shows the increased requirements for even small improvements in drag and so small geometry changes which alter the flow field and body forces are of increased interest.

For SUVs, their blunt rear geometries are less than ideal when considering aerodynamic characteristics. The blunt trailing edge associated with this type of vehicle means a large proportion of the overall drag, around 30\% [3], can be attributed to base pressure. This is due to the separation induced by the geometry causing a large low pressure wake. The wake is often characterised as a time-averaged toroidal structure enclosed by the free shear layers emanating from the roof, sides and under-body [4]. However, in practice this structure is rarely present when time-dependent flow fields are considered. Instantaneous images of the flow on the symmetry plane within the wake show that the main recirculating structures, known to form the arms of the time-averaged toroid, are still present but in a less defined form and, they are accompanied by many smaller vortical structures that shed from the trailing edges of the model [5]. The positions of the recirculating vortex cores can also be seen to vary with time as the relative magnitudes of the two recirculating structures in a given plane alternate; often linked to a short time scale flapping of the wake driven by von Kármán shedding or, if certain conditions facilitate it, longer time scale bi-stable switching $[6,7,8]$. Despite the time-averaged vortex ring not being present when an instantaneous snapshot of the wake is considered it is still directly linked to the base pressure distribution. Therefore previous attempts to modify the instantaneous wake topology to such an extent that the time-averaged toroid, and so base pressure, are also altered has been seen to result in significant drag reductions.

\subsection{Passive and Active Base Drag Reduction Methods}

Passive methods are capable of reducing the effect of the recirculating structures the base surface by moving the toroid downstream. Base cavities [9, 10, 11], splitter plates [12] and extension plates [13] have all been successful in reducing drag by increasing the distance between the base surface and the recirculation.. As well as, in the case of cavities and extension plates, increased pressure recovery due to the angled trailing edge surfaces that 
enable increased flow attachment. Drag reduction by pressure recovery is also observed in the case of side and/or roof tapering [14, 15, 16] and boat-tailing [17]. All these studies achieve a respectable drag reduction in their optimal configuration, however for SUV geometries in particular they have limitations. Cavities and plates would cause issues for vehicle aesthetics, whilst tapering or boat-tailing removes the blunt silhouette often favoured for SUV vehicles resulting in only small angles being applied. Therefore a physically smaller modification would be preferred.

Active drag reduction methods are initially attractive because such devices could be implemented with little effect on the appearance of the vehicle; an important styling consideration. Examples include increasing the base pressure using base bleed [18], steady [19] and pulsed jets [20] which have shown drag reductions, but in practice these are partially offset once the power requirements of the device are considered. However, understanding the mechanisms of drag reduction from an active device may lead to ways to replicate these effects in a passive way. An example of this is steady blowing at the upper trailing edge of a simplified model [19]. With a jet angled into the wake a drag reduction was observed due to two factors. The first being reduced wake size and the second reduced strength of the lower recirculation and so reduced near wall velocity.

\subsection{Application of Base Slats}

In an attempt to replicate the disruption to the lower recirculation seen with the steady blowing, Littlewood et al. [21] applied small horizontal slats to the base of a quarter scale simplified reference body, known as the Windsor model [22], which has a more representative shape than the commonly used Ahmed geometry [23]. Wind tunnel tests were performed at three different wind speeds, all of which correspond to representative Reynolds numbers. The best tested configuration placed 4 equally spaced slats on the lower half of the model base, with each slat being $1 \mathrm{~mm}$ thick and extending $8 \mathrm{~mm}$, or $0.8 \%$ of the model length, into the wake. This configuration resulted in the drag coefficient reducing by 0.008 (often referred to as 8 counts). The source of drag reduction was explained by considering pressure measurements obtained using an array of 111 pressure tappings applied to the base of the model. These showed an increased pressure region directly above the highest slat from which it was inferred that the lower recirculation impinged on the upper surface of this slat. Additionally a reduction in the suction region at the centre of the lower vortex was also observed. Although no wake data was obtained it is suggested the pressure changes seen are due to a reduction of the rotational energy of the lower recirculation. Littlewood [21] goes on to report full scale tests that demonstrated the need for a smooth under-body for the slats 
to be effective, highlighting that the mechanism of drag reduction is primarily attributed to the modification of the lower recirculation within the wake.

Robertson et al. [24] aimed to gain a deeper understanding of the mechanisms of drag reduction from the slats by completing a computational study of the same geometry. As seen experimentally, a high pressure region above the slat improved base pressure and wake analysis showed a reduced wake length. This was attributed to a change in wake balance as increased turbulent kinetic energy was seen for the upper vortex indicating a higher level of energy dispersion responsible for the reduced wake size. However, the study employed steady-state Reynolds-Averaged Navier Stokes (RANS) which is generally accepted to be insufficient for this type of geometry. Typically only the drag force can be well replicated with the generated wake structures and pressure distributions often being incorrect [25]. This is due to the differences between the instantaneous and time-averaged flow structures defined previously, with a steady computational approach failing to capture the time-dependent physics such as vortex shedding and wake flapping found within the flow. The inclusion of these time-dependent flow features is seen to change the time-averaged result of an unsteady simulation, indicating the replication of these physics drives the production of a correct timeaveraged flow field.

\subsection{Scope of the Paper}

Despite the limitations of the published work, they highlight the potential that quite small geometry changes can alter the wake and drag of a simplified vehicle. Therefore a more in-depth computational investigation has been completed, this time considering a single horizontal slat at different locations on the base of a simplified vehicle. For this work an unsteady methodology was applied to enable the effects of the slat on the instantaneous flow structures to be established whilst also producing a more representative time-averaged flow field than that seen by Robertson et al. [24]. Understanding wake sensitivity to this relatively small modification could help inform future design work. For example, the location of a rear screen wiper might be optimised to reduce the vehicle drag whilst ensuring that other detrimental global changes are not introduced.

This paper is organised into two main sections, the first presents an experimental validation of the CFD methodology. This is followed by a thorough analysis of the flow field, drag and lift for each slat configuration. These results are discussed in detail to enable a description of the flow mechanisms to be provided and for these flow mechanisms to be linked to the base pressure and force changes that are seen. This thorough approach allows the effects and mechanisms of the geometry modification to be explained whilst also providing 


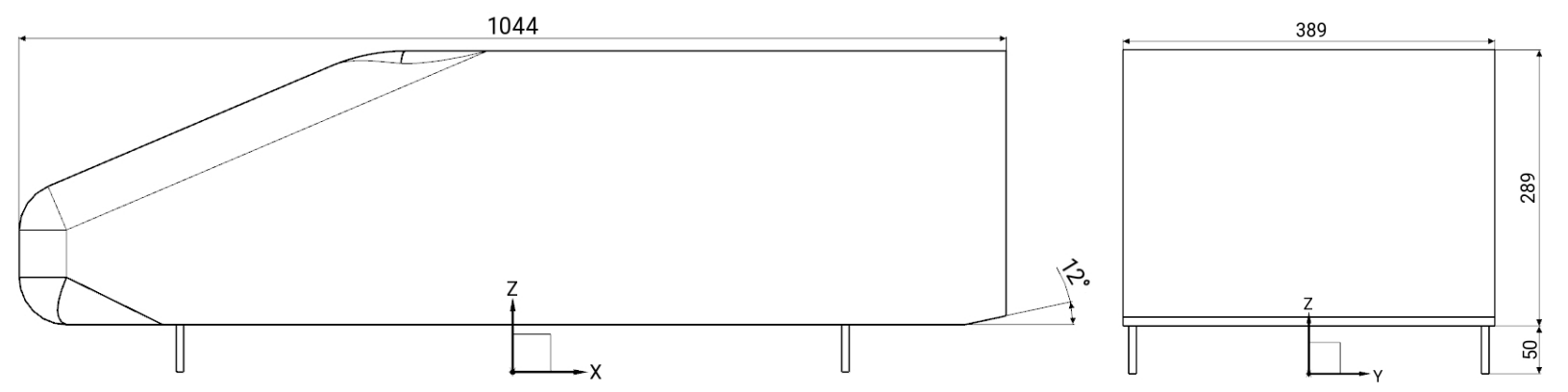

Figure 1: Windsor model with $12^{\circ}$ taper on the lower base edge, all dimensions in mm.

a useful insight into how small near wall structures interact with bulk flow features. Finally, conclusions and suggestions for further work are given.

\section{Methodology}

\subsection{Model Selection}

For this investigation the Windsor model variant shown in Figure 1 was used. The standard dimensions of Length $(\mathrm{L})=1.044 \mathrm{~m}$, Width $(\mathrm{W})=0.389 \mathrm{~m}$ and Height $(\mathrm{H})=0.289 \mathrm{~m}$ are used, making the model equivalent to a $1 / 4$ scale passenger car. To prevent separation the front radii are $0.05 \mathrm{~m}$ and the roof has a $0.2 \mathrm{~m}$ radius. This slanted front-end geometry generates a more representative flow and so makes the model more favourable than a traditional Ahmed geometry [23]. For this study the origin was taken to be on the ground plane at the model centre as illustrated in Figure 1.

Rather than use the true squareback configuration a $12^{\circ}$ lower taper with a length of $45 \mathrm{~mm}$ was added in order to reduce the effect of bi-stability known to be present in the wake of the squareback model [15]. This, in turn, reduces the required period of data collection which is necessary for a computational study as the time required to produce a true average field for the squareback model was seen to be in the region of 630 seconds experimentally, which is not feasible with the current computational resources. The effect of adding a lower edge taper has been previously studied experimentally and is well documented by Perry et al. $[26,14]$ and Pavia et al. [15]. The main changes to the flow topology are due to the upwash induced by the tapered surface. This increased upwash modifies the balance between the upper and lower recirculations, reducing the level of symmetry compared to the square-back configuration. Higher momentum flow is now being fed into the lower recirculation increasing its size - resulting in the base impingement being moved toward to upper trailing edge. This corresponds to the changes observed in base pressure, the topology of the distribution is now 

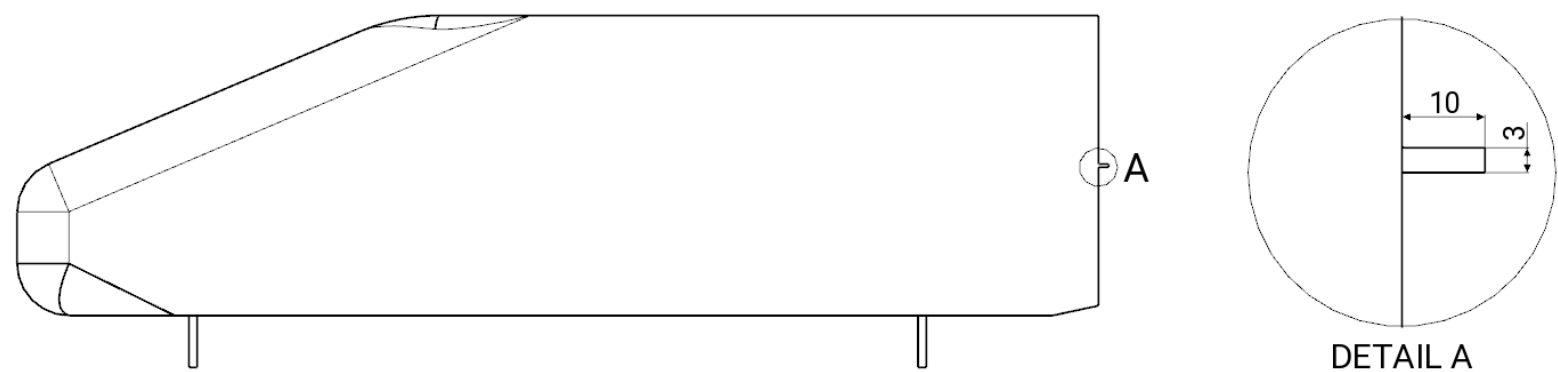

Figure 2: Detail view of the slat geometry with dimensions in $\mathrm{mm}$, illustrated in the mid-base slat configuration.

a ' $U$ ' shaped low pressure region on the lower portion of the base surface. Another result of the increased upwash is a reduced wake length due to the angle at which the under-body flow enters the wake. A further consequence being the saddle point at which the wake closes being moved upward. When considering the body forces a reduction in lift is seen along with increased drag. This is expected given the lower edge taper acts as a diffuser, a device which is often used to increase the downforce of a body with minimal drag penalty [27]. The addition of the taper also makes the model more comparable to real world geometries as most practical vehicles will not have a square rear lower side profile.

\subsection{Slat Configurations}

The slat dimensions are illustrated in Figure 2, each slat is $3 \mathrm{~mm}$ thick and extends $10 \mathrm{~mm}$, or $1 \%$ model length, into the wake. To further illustrate how small the geometry modification is when given in terms of model height the slat's dimensions are $0.01 \mathrm{H}$ wide and $0.035 \mathrm{H}$ long. These dimensions were chosen based on the previous experimental slat study [21], whilst finding a balance between manufacturability for experimental validation purposes and a desire to keep them as small as possible for design purposes.

The slat locations considered for this study, referred to as $S_{z}^{*}$, are normalised by model height, $\mathrm{H}$, and measured from the underside of the model. (Table 1) gives the tested configurations, which are also illustrated in Figure 3. The positioning of the slats was determined by considering the previous studies and knowledge of the baseline flow field. In Littlewood's work the greatest drag reduction was achieved when four slats were equally spaced over the lower half of the model base, placing the highest slat at mid-base height [21]. As the largest changes in base pressure were seen in close proximity to this slat it was defined as the first configuration $\left(\mathrm{S}_{\mathrm{z}}^{*}=0.5\right)$. Also, in this previous configuration, base pressure increases were seen over the entire area above the slat. Therefore, moving the slat down, to $\mathrm{S}_{\mathrm{z}}^{*}=0.375$, 
Table 1: Definition of each slat location $\left(S_{z}^{*}\right)$, normalised by model height and measured from the underside of the model.

\begin{tabular}{|l|c|c|}
\hline Slat Configuration & Slat Location $\left(S_{z}^{*}\right)$ & Colour \\
\hline Lower-Slat & 0.375 & Aqua \\
Mid-Base Slat & 0.5 & Red \\
Upper-Slat & 0.625 & Purple \\
Upper-Quarter Slat & 0.75 & Orange \\
\hline
\end{tabular}

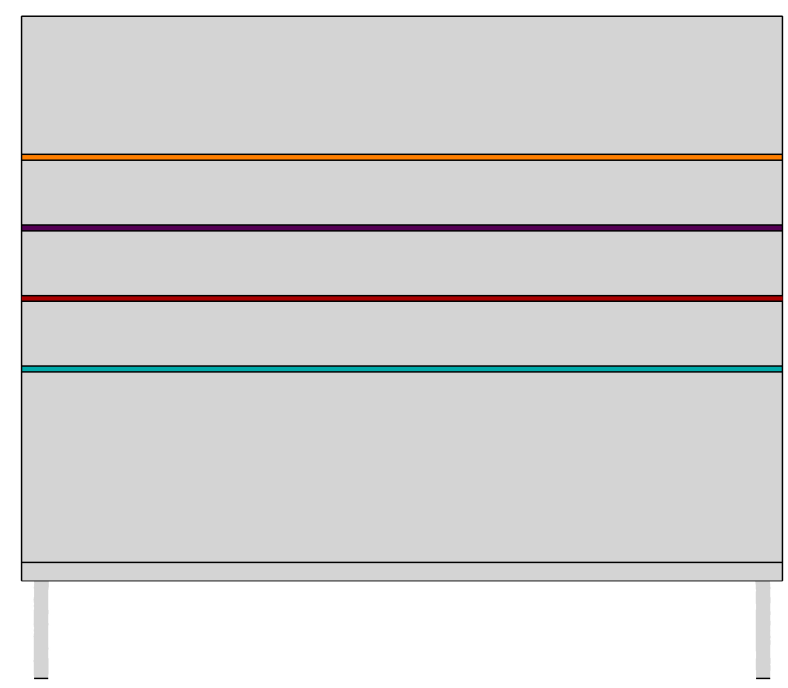

Figure 3: Illustration of all four slat locations; lower-slat (aqua), mid-base slat (red), upper-slat (purple) \& upper-quarter slat (orange).

西

would increase the base area above the slat over which the increased pressure acts resulting in a greater drag reduction. Finally, it was of interest to consider how the slat's proximity to the base impingement altered the result. As Littlewood [21] concluded the recirculating flow's impingement on the upper slat surface was the cause of the pressure increase the slat was moved closer to the location of the base impingement to maximise the stagnation effect. When considering the baseline flow field the base impingement was observed at approximately three-quarters of the base height and so slats were placed at equal intervals to give the upper $\left(\mathrm{S}_{\mathrm{z}}^{*}=0.625\right)$ and upper-quarter $\left(\mathrm{S}_{\mathrm{z}}^{*}=0.75\right)$ slats.

\subsection{Computational Setup}

The Windsor model is known to produce a three-dimensional and highly unsteady wake meaning steady approaches such as RANS are insufficient at capturing the required timedependent turbulent structures to fully replicate the flow field [28]. Therefore an unsteady approach, such as Large Eddy Simulation (LES), is required however due to high resolution 
mesh requirements it comes with a large computational cost. This computational expense can be reduced by considering a hybrid RANS-LES model such as DES, first suggested by Spalart et al. [29]. RANS is used in the near-wall regions allowing a reduced local grid size, whilst away from the wall LES is employed ensuring the unsteady turbulent structures are resolved. A blending factor, based on grid size, dictates where the switch between models occurs as the turbulent length scales resolved are dependent on cell size within the LES model. DES is known to perform well for flows with a large separated region, where the point of separation is dictated by the geometry and so is insensitive to the near-wall flow as this ensures a near-wall RANS approach will be sufficient. For the Windsor model it is known that the separation is induced by the sharp trailing edges, meaning DES can be implemented with a high level of confidence [30]; it has consequently been used in its different variations for many automotive applications [31, 32, 33].

Within this work Improved Delayed Detached Eddy Simulation (IDDES) has been used with a k-omega SST near wall treatment. The IDDES model deals with two issues faced by the traditional DES approach; grid induced separation and log layer mismatch. The first of these was observed by Menter et al. [34] who identified that high near-wall grid resolution could cause a premature switch from RANS to LES resulting in flow separation. They solved this problem by adapting the blending function within the SST model to delay the transition. Spalart et al. [35] furthered this to develop a generic shielding function used to delay this separation by considering both eddy viscosity and wall distance, making it applicable to any implemented eddy viscosity based DES model. This model, Delayed Detached Eddy Simulation (DDES), has effectively superceded the traditional DES approach. Despite this it still suffers from log layer mismatch, whereby the intercept of the log law region found at the interface of the RANS and LES regions do not match. Shur et al. [36] suggested the IDDES model which increases the resolved near-wall turbulence resulting in a better matched interface. Although more complex, IDDES has a wider range of applications whilst matching or surpassing the performance of DDES making it the model of choice for this work.

The computational domain (Figure 4) was defined to reflect the wind tunnel test conditions under which the validation data would be obtained. This means the model was placed within a constant rectangular cross section domain $1.92 \mathrm{~m}$ wide by $1.32 \mathrm{~m}$ high to reflect a simplified version of the tunnel's working section. The inlet length of the domain, 15H, was chosen so the boundary layer at the front of the model matched that measured in the experimental case, with a displacement thickness of $7.2 \mathrm{~mm}$. The inlet velocity was $40 \mathrm{~m} / \mathrm{s}$ 


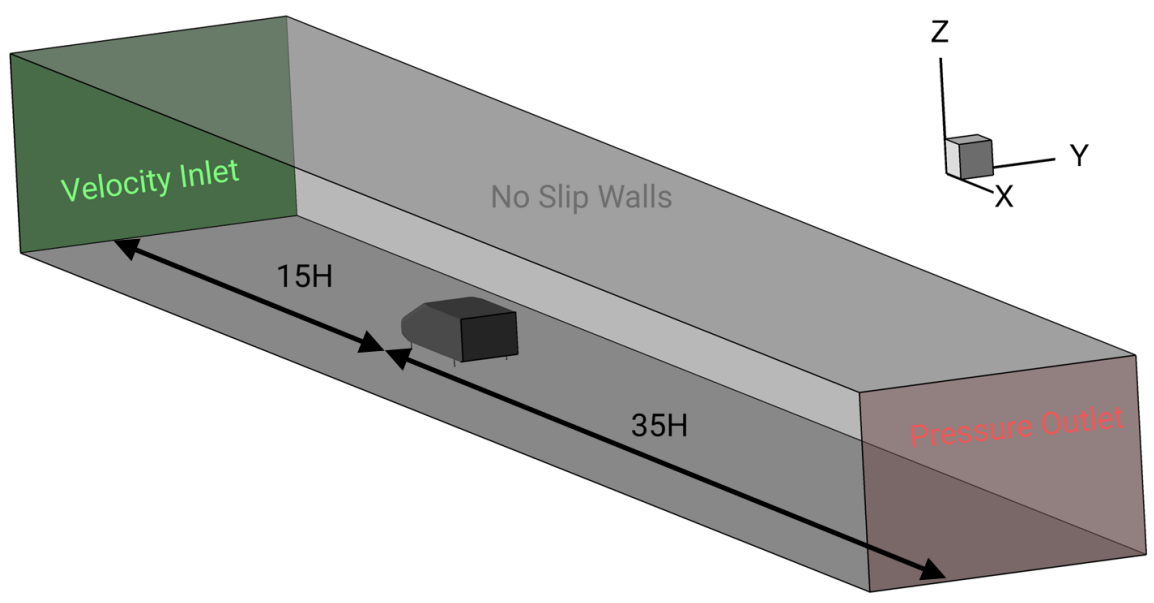

Figure 4: Illustration of the computational domain.

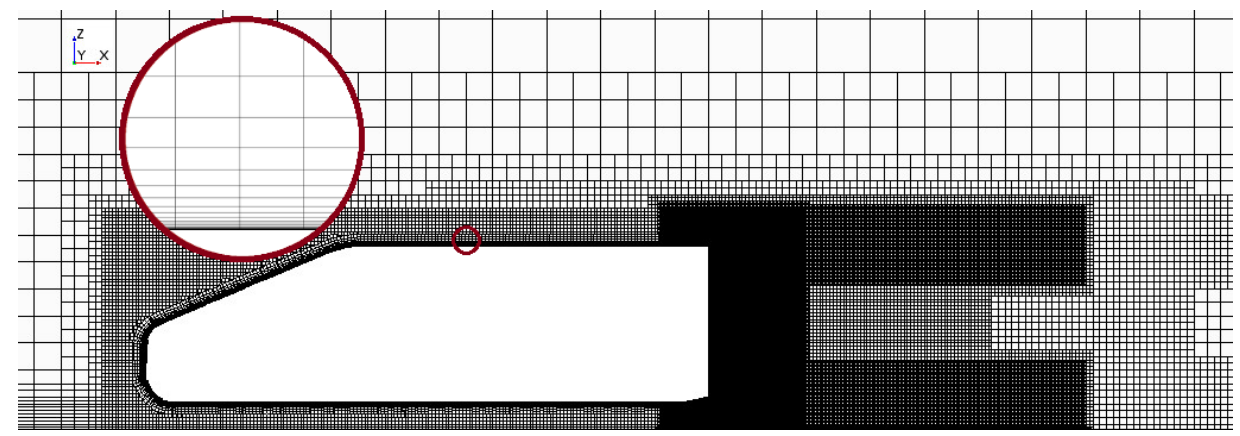

Figure 5: Mesh around model, including detailed view of prism layers, taken from the $\mathrm{y}=0$ mid-plane.

with a turbulence intensity of $0.2 \%$ and the model was positioned at a ground clearance of $0.05 \mathrm{~m}$. The domain was discretised using a Cartesian mesh with prismatic layers adjacent to walls as illustrated in Figure 5. For the prism layers, the first cell size is defined as $5 \times 10^{-7} \mathrm{~m}$ which results in a wall $y^{+}<1$ ensuring the boundary layer on the model is resolved rather than modelled. Refinement was focused in the regions of interest and the mesh density decreased away from the model where the relative gradients within the flow were reduced. Applying this meshing strategy gave a cell count of around 22 million cells which compares well to similar studies [17]. A mesh sensitivity study was completed and justified this mesh as doubling the cell count achieved a negligible drag change of $2 \%$.

The characteristic time $\left(\mathrm{t}^{*}\right)$ is defined as the time taken for the flow to travel the characteristic distance, which in this case is the model height. The time step $(\Delta \mathrm{t})$ of $3.612 \times 10^{-5}$ seconds results in 200 time steps per $t^{*}$. Based on this time step the Courant-Friedrichs- 


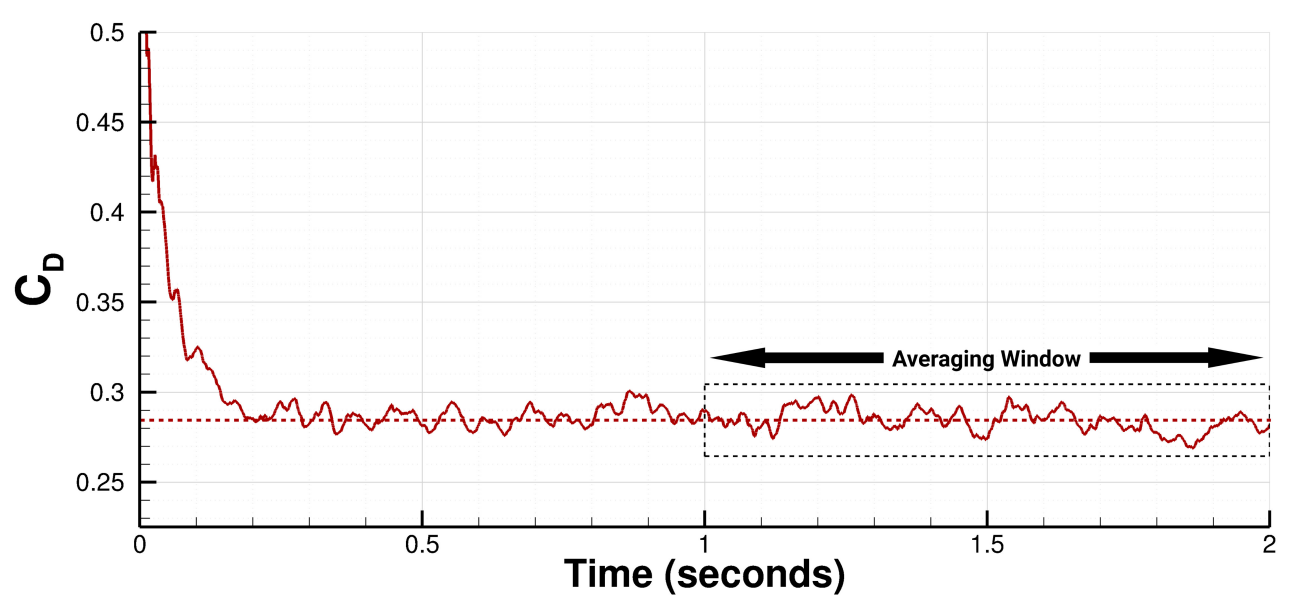

Figure 6: Example drag coefficient history, with averaging window indicated by dashed box.

Lewy (CFL) number was evaluated and found to be in the region of 1 around the model. Sterken et al. [37] found a similar result and justified this value by halving the time step to ensure $\mathrm{CFL}<1$, with this condition a negligible change was observed in all measured forces meaning the reduction in CFL was not worth the additional computational cost. Figure 6 illustrates how the drag coefficient develops with time from initialisation of the simulation. To ensure a fully-developed, quasi-steady state had been reached an initial settling period of 1 second was defined. Given the results shown in Figure 6 an initialisation period of 0.5 seconds appears to have been sufficient indicating computational costs could be reduced in further work. This couldn't be implemented within this study as the initialisation period and averaging window had to be pre-defined. Forbes et al. performed a computational study on a simplified two-box model, known as the generic SUV model developed by Wood et al. to replicate market trends in consumer SUV geometries [3]. The study implemented an averaging period of 1 second and achieved well-validated CFD results. Given that this model is also quarter scale, the same averaging window was applied in this work as it has been seen to allow a large enough number of flow passes, $138 \mathrm{t}^{*}$, to produce the required flow features.

\subsection{Experimental Setup}

All experimental testing was carried out in Loughborough University's Large Wind Tunnel illustrated in Figure 7 and described by Johl [39]. This is an open-loop tunnel with a working section of $2.5 \mathrm{~m}^{2}$, giving a blockage ratio of $4.4 \%$ with this Windsor model in place. The model is supported by four M8 bars at a ground clearance of $0.05 \mathrm{~m}$ above the fixed 


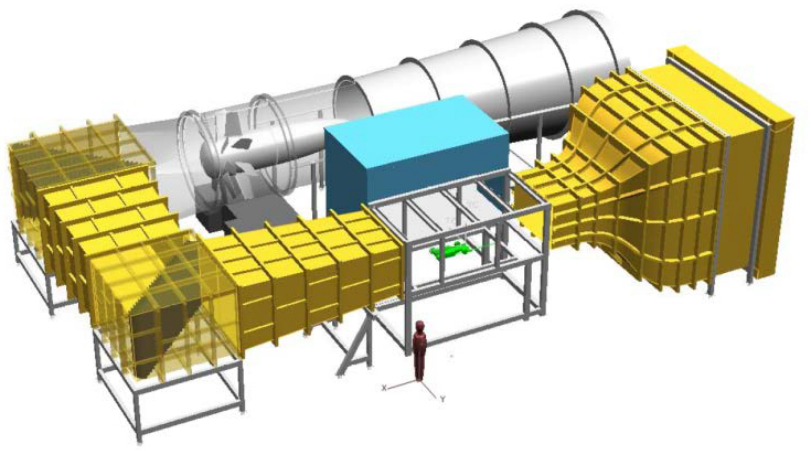

Figure 7: The Loughborough University wind tunnel [38].

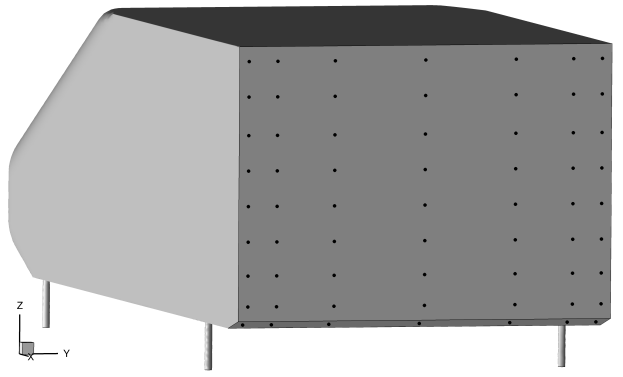

Figure 8: Pressure tapping distribution over the base surface and lower taper of the model. 
ground plane. No correction has been applied to the forces to account for these bars. The flow velocity was set to $40 \mathrm{~m} / \mathrm{s}$ with a free-stream turbulence of $0.2 \%$ and flow uniformity of $\pm 0.4 \%$.

The base and slant surface were populated with a grid of pressure tappings spread across the entire width of the model, as is it is known the instantaneous base pressure distribution is frequently asymmetric. 56 and 7 tappings were used for the base and slant surfaces respectively (Figure 8). The area toward the model edges was more densely populated to account for the higher pressure gradients expected in this region. Pressure measurements were collected over a 600 second period at a sampling rate of $260 \mathrm{~Hz}$ with the accuracy of the pressure scanner being $0.06 \%-0.1 \%$ of full scale $( \pm 2.2 \mathrm{kPa})$ depending on the operating conditions. Pressure coefficients were calculated using Equation 1 considering the recorded pressure $(\mathrm{p})$ along with the free-stream static pressure $\left(\mathrm{p}_{\infty}\right)$ and free-stream velocity $\left(\mathrm{V}_{\infty}\right)$ both measured using a Pitot-static tube placed upstream of the model. The air temperature was also recorded and used to calculate air density $(\rho)$. All experimental and computational pressure measurements were blockage corrected using Equation 2 [40].

$$
\begin{gathered}
C_{P}=\frac{p-p_{\infty}}{0.5 \cdot \rho \cdot V_{\infty}^{2}} \\
C_{P_{\text {corr }}}=\frac{C_{P}+2 \frac{A_{m}}{A_{t}}}{1+2 \frac{A_{m}}{A_{t}}}
\end{gathered}
$$

where $C_{P_{\text {corr }}}$ is the corrected surface pressure coefficient, $C_{P}$ is the measured surface pressure coefficient, $A_{m}$ is the cross-sectional area of the model and $A_{t}$ is the cross-sectional area of the tunnel working section.

Balance measurements were obtained using a six component underfloor balance, with an accuracy of $0.01 \%$ of full scale for drag $( \pm 120 \mathrm{~N})$. Data was collected at $300 \mathrm{~Hz}$ for 600 seconds after an initial settling period. Each measured force $(\mathrm{F})$ has been non-dimensionalised using using Equation 3, which uses a corrected velocity ( $\mathrm{u}_{\text {corr }}$ ) estimated via the continuity correction defined in Equation 4 [41]. This same correction has also been applied to the computational result.

$$
\begin{gathered}
C_{\text {force }}=\frac{2 F}{\rho \cdot u_{\text {corr }}{ }^{2} \cdot A_{m}} \\
u_{\text {corr }}=\frac{V_{\infty} \cdot A_{t}}{A_{t}-A_{m}}
\end{gathered}
$$




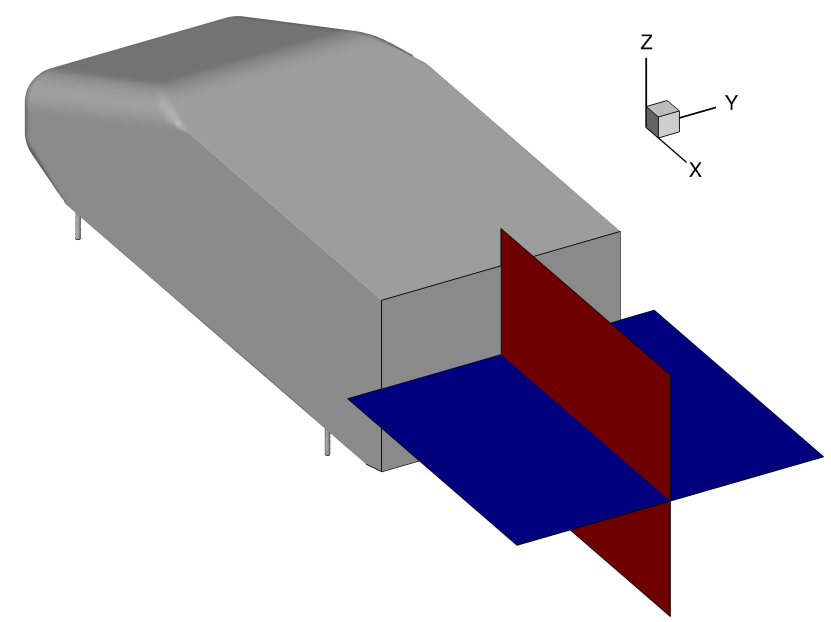

Figure 9: The locations of the two PIV planes used for wake visualisation; vertical mid-plane in red and horizontal mid-plane in blue

Particle Image Velocimetry (PIV) was used to obtain two-dimensional, two component, planar velocity fields in the vertical and horizontal wake mid-planes; the locations of which are illustrated in Figure 9. The PIV was performed using a 200mJ double pulse Nd:YAG laser with the flow seeded with $1 \mu \mathrm{m}$ DEHS (Di-Ethyl-Hexyl-Sebacat) particles. Two 4 megapixel LaVision Imager ProX cameras were used with $50 \mathrm{~mm}$ lenses resulting in an approximate resolution of 5 pixels per $\mathrm{mm}$ for each of the cameras. The cameras were located side by side in the stream-wise direction to capture the entire wake length at a higher resolution with an appropriate overlapping field of view. Both the cameras and the laser were triggered using a programmable timing unit controlled using commercially available DaVis software at $7.26 \mathrm{~Hz}$, the maximum recording frequency of the cameras.

The images were pre-processed using a minimum background subtraction over all of the images. The processing initially used 128x128 pixel windows with a $50 \%$ overlap decreasing in size to the final window size of $24 \times 24$ pixels with a $50 \%$ overlap. The final window size was used for two passes and applied a circular weighting to the windows. All processing was completed using the aforementioned DaVis software. For this set-up the level of uncertainty in the velocity measurements can be estimated at $0.5 \%$ of the mean and $1.5 \%$ of of the Root Mean Square (RMS) values in the free-stream [6].

\section{Experimental Validation}

In order to validate the $C F D$ methodology the baseline and mid-base $\left(S_{z}^{*}=0.5\right)$ slat configurations were simulated and results compared to experimental data. The two quanti- 
tative comparison metrics will be the drag coefficient $\left(\mathrm{C}_{\mathrm{D}}\right)$ and base pressure drag coefficient $\left(\mathrm{C}_{\text {Dbase }}\right)$; which is found by integrating the surface pressures over the base surface area (A) as defined in Equation 5.

$$
C_{\text {Dbase }}=\frac{1}{A} \int C_{P} \cdot d A \approx \frac{1}{A} \sum_{i=1}^{N} C_{P} \cdot A_{i}
$$

where $\mathrm{N}$ is the total number of pressure tappings and $A_{i}$ is the projected base surface area associated to a given pressure tapping. Base pressure distributions and wake midplane visualisation will also be used to validate the baseline configuration, with vertical and horizontal mid-planes considered. For the mid-base $\left(\mathrm{S}_{\mathrm{z}}^{*}=0.5\right)$ slat configuration just forces and base pressures were considered. These were deemed to be sufficient as it would be hard to obtain good quality PIV data sufficiently close to the base and slat surfaces experimentally to validate the computational result here.

Throughout the paper normalised quantities have been presented and are denoted with a * The reference values used are model height, $H$, and free-stream velocity, $V_{\infty}$. To improve the communication of the results within the wake, figures showing this region define the base surface as $x^{*}=0$. This allows the origin to remain at mid-wheelbase, as dictated by convention, whilst also enabling easier interpretation of the wake length.

\subsection{Baseline Configuration}

Table 2 shows the experimental and computational drag coefficients along with base pressure drag coefficients. There is a small difference of approximately $8 \%$ in drag coefficient, whereby the computational result under-predicts drag. However, the base pressure drag is very well matched with a difference of just $2 \%$ between the computational and experimental results. This indicates the CFD is under-predicting a source of drag elsewhere, however as the experimental result is limited to overall body drag and base pressure tappings this source cannot be easily identified. This difference is unlikely to impact the simulation's ability to predict the changes due to the addition of a slat as the flow changes are expected to occur in the base region, where the result is well predicted.

The high contribution of base pressure drag to the overall drag is typical for this type of geometry due to the large separated wake region found behind the model. Here the wake structure is characterised and validated by considering the time-averaged flow field. The averaged wake consists of a toroid formed as flow rolls over each of the model edges, consistent with similar geometries reported in the literature $[42,26]$. Due to the lower taper present in this case the ring vortex is distorted, with the lower recirculation dominating, as 
Table 2: Mean values for computational and experimental drag coefficient and base pressure drag coefficient for the baseline and mid-base slat configurations.

\begin{tabular}{|c|c|c|}
\hline & Experiment & CFD \\
\hline Baseline $C_{D}$ & 0.291 & 0.267 \\
Baseline $C_{\text {Dbase }}$ & 0.173 & 0.177 \\
Mid-Base Slat $C_{D}$ & 0.292 & 0.268 \\
Mid-Base Slat $C_{\text {Dbase }}$ & 0.181 & 0.180 \\
\hline
\end{tabular}

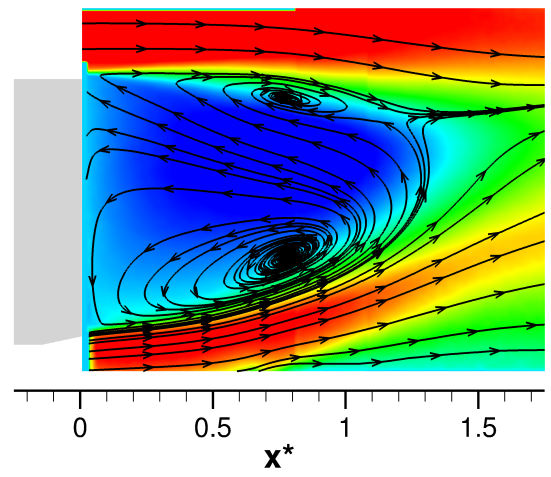

(a) Experimental Data

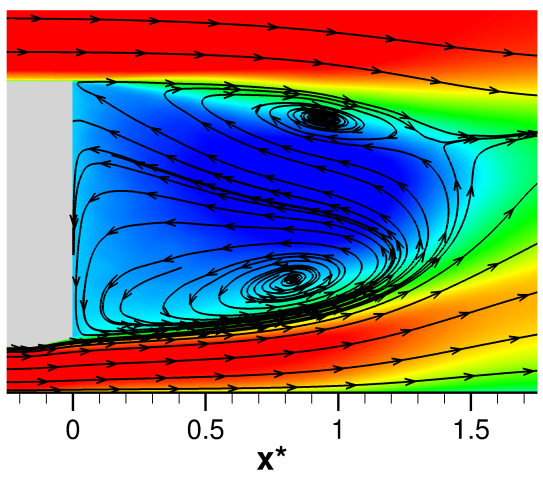

(b) Computational Data

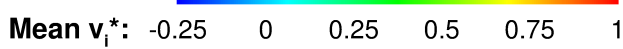

Figure 10: Time-averaged vertical mid-plane $(y=0)$ within the wake for the baseline configuration. $\mathrm{x}^{*}=0$ has been defined as the location of the model base surface to enable easier interpretation of the result.

shown in Figure 10. This can be attributed to the lower taper accelerating the flow under the model into the wake, whilst also angling the flow toward the center.

This dominating lower recirculation is seen to be one of the main sources of drag, when considering the base pressure distribution in Figure 11. To improve the quality of the comparison the locations of the 56 experimental base pressure tappings (Figure 11a) have been used to extract data from the computational result (Figure 11b) to enable the distributions to be obtained using the same spatial resolution and locations. This is important as the reduced experimental resolution results in less well described pressure gradients over the base surface. This would result in the introduction of errors in the base pressure drag calculation if the full resolution of the computational data set (Figure 12) was compared to the experimental result. Applying this method also ensures the same area of the base is considered, highlighting a benefit of the computational data set. The experimental result can only be interpolated within the bounds of the pressure tapping locations, however com- 


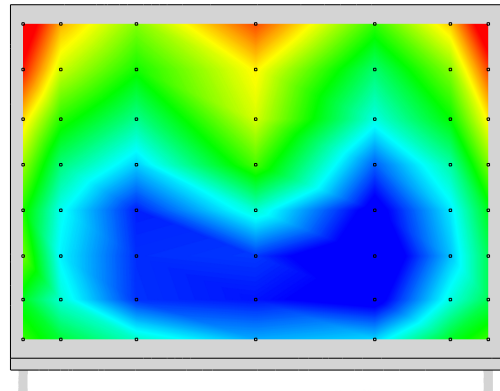

(a) Experimental Data

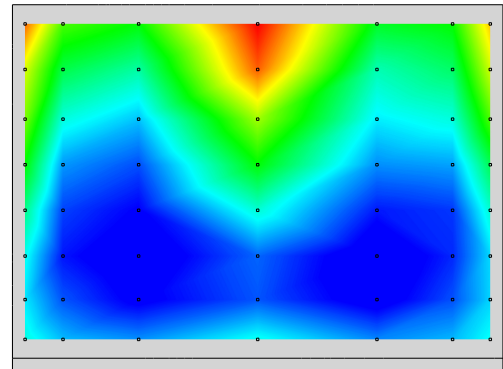

(b) Computational Data

Mean $\mathrm{C}_{\mathrm{p}:}-0.19 \quad-0.18 \quad-0.17 \quad-0.16 \quad-0.15 \quad-0.14$

Figure 11: Time-averaged base pressure distribution for the baseline configuration, with the pressure tapping locations indicated.
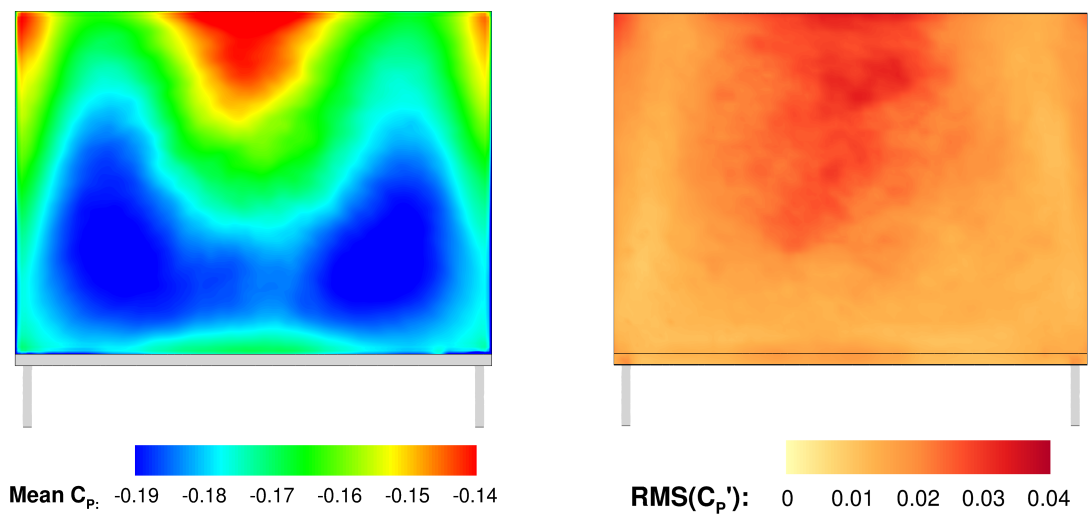

Figure 12: Time-averaged base pressure distribution for the baseline configuration at the full computational resolution (left). Root mean square of the pressure fluctuations over the base surface for the full resolution computational result (right). 
putational data can be collected over every model surface, with limited interpolation due to the increased resolution of data points. Inconsistent measurement areas between numerical and experimental data sets would also introduce errors within the validation. These two factors should not be overlooked when comparing two data sets, especially those obtained using different methods.

The regions of lowest pressure can be found on the lower portion of the base, at approximately the height of the lower recirculation. The region of highest pressure is found at the impingement point of the lower recirculation, at approximately $3 / 4$ of the base height. This agrees with Grandemange et al. [43] who also found that for a wake dominated by the lower recirculation, an impingement above the mid-base height of the model was observed and accompanied by a positive pressure gradient along $\mathrm{y}=0$ on the base surface. The RMS of the fluctuation in the base pressure coefficient (Figure 12) shows the highest level of fluctuation is found toward the upper trailing edge of the base. This is similar to the results seen by Pavia et al. [15] who found the addition of a lower taper moved the fluctuations upwards compared to a squareback model. This figure also shows that there is only one distinctive region of high RMS, indicating no bi-stability is present in the computational result. Therefore, given the sampling time considered and the symmetry of the model, the wake is expected to be symmetric in the horizontal mid-plane as illustrated in Figure 13.

Throughout Figures 10-13 the computational and experimental flow features and base pressures observed are all in good agreement in terms of both wake structures and the magnitude of the variables displayed. The main difference observed is in the region of the lower taper where the experimental result sees a higher degree of upwash. The result of this is a shorter recirculation length and a more angled wake along with a separation on the tunnel floor. Despite these differences the near wake flow is well replicated, in particular the angle of the return flow, the location of base impingement and the near wall flow velocities. This explains why the base pressure distributions are still so well matched, even though the wakes have visible differences. The distributions themselves are reasonably similar in shape and magnitude, with the lowest pressure region being slightly larger in the computational result. This effect is exaggerated by extracting data only at the experimental resolution, if the full data set is considered the error is reduced from $2 \%$ to $0.5 \%$, highlighting the data loss when considering a reduced spatial resolution.

\subsection{Mid-Base Slat Configuration}

To prove the robustness of the CFD methodology further one of the slat configurations (mid-base $\left(\mathrm{S}_{\mathrm{z}}^{*}=0.5\right)$ slat) was also tested experimentally. The effect of the addition of the 


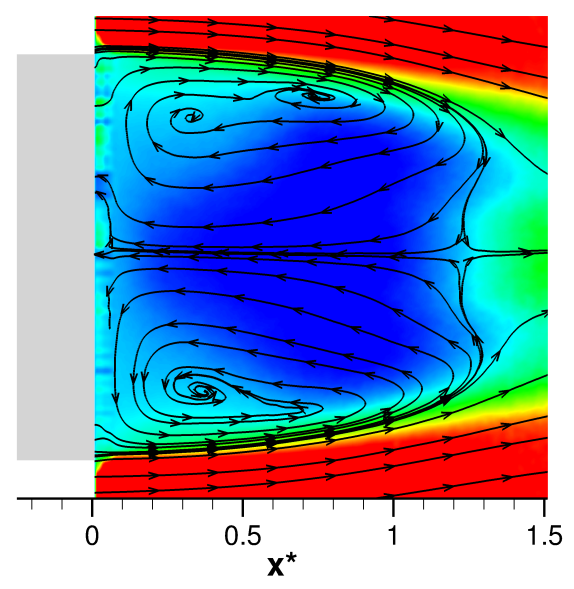

(a) Experimental Data

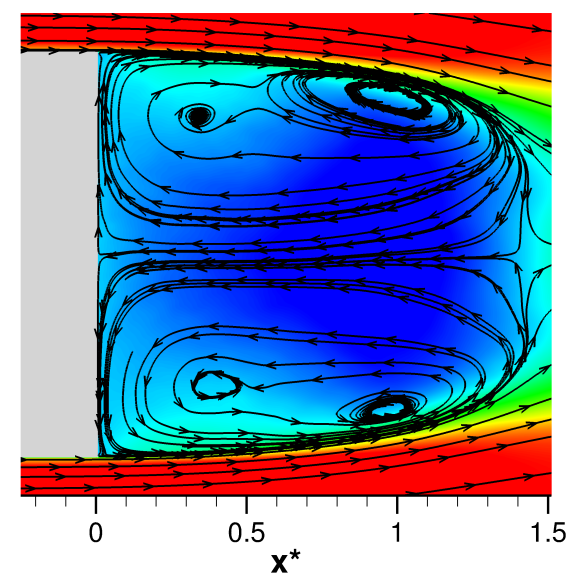

(b) Computational Data

Mean $\mathbf{v}_{\mathrm{i}}^{*}: \begin{array}{rlllll}-0.25 & 0 & 0.25 & 0.5 & 0.75 & 1\end{array}$

Figure 13: Time-averaged horizontal mid-plane $\left(\mathrm{z}^{*}=0.67\right)$ within the wake for the baseline configuration. $\mathrm{x}^{*}=0$ has been defined as the location of the model base to enable easier interpretation of the result.

slat on the flow field will be discussed in detail within the subsequent section, meaning here only the validation will be discussed. The overall body drag for the mid-base $\left(\mathrm{S}_{z}^{*}=0.5\right)$ slat configuration is again under-predicted in the computational result with the percentage difference remaining $8 \%$ as it was for the baseline case. This means the change in model drag due to the addition of the slat is accurately replicated.

Base pressure measurements were also taken and the distributions shown in Figure 14 were obtained using the same methodology as outlined in Section 3.1. Once again the need for matching the resolution for comparison purposes is highlighted when the limited base area obtained using the experimental resolution is considered. The base pressure distribution shows good agreement, once again indicating the flow features are well replicated. This is further illustrated when considering the base pressure drag coefficient which is replicated by the numerical result to within $1 \%$ of the experimental result. PIV data was not collected for this configuration as it would be difficult to obtain high quality data in the regions of interest, near the base and slat surfaces. Despite this, the validation data presented here was deemed sufficient due to the high level of agreement in the metrics related to the base surface. 


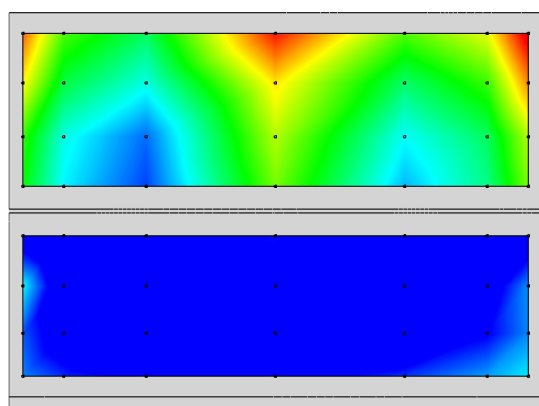

(a) Experimental Data
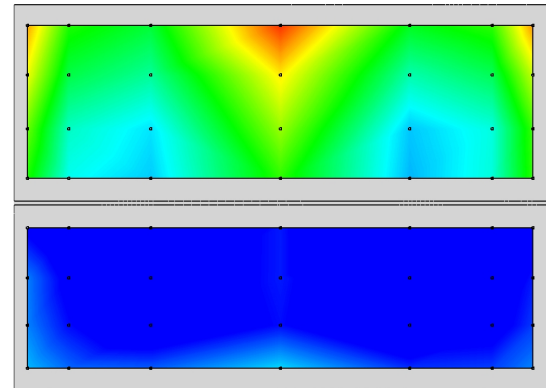

(b) Computational Data

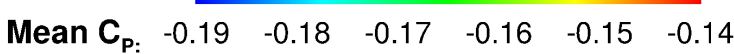

Figure 14: Time-averaged base pressure distribution for the mid-base slat configuration, with the pressure tapping locations indicated.

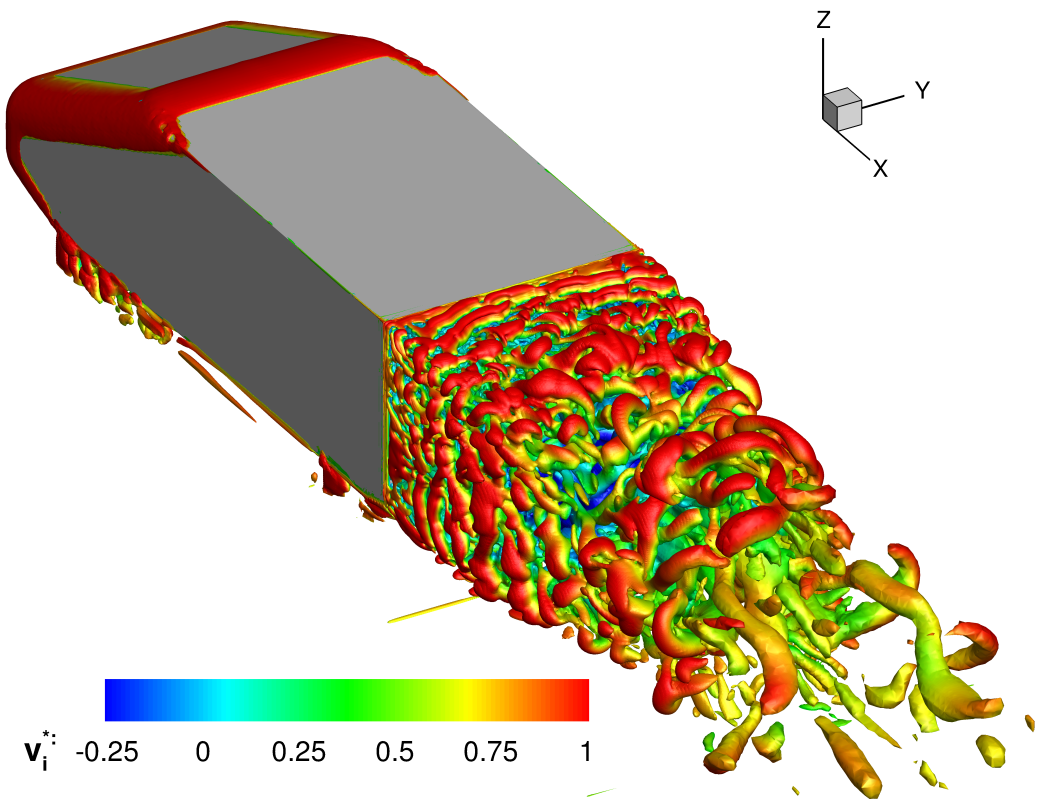

Figure 15: Instantaneous vortical structures within the wake as illustrated via an isosurface of normalised Q-criterion $\left(\mathrm{Q}^{*}=5\right)$. 


\subsection{Validation Findings}

The CFD methodology has been well validated by experimental data specifically global forces, base pressures and wake planes. This has been achieved in part by ensuring the CFD conditions, such as inlet boundary conditions and tunnel dimensions, replicated those of the experimental setup. The high level of confidence in the computational result enables a more thorough analysis of the flow to be completed through use of the more highly resolved CFD data set. The full resolution of the computational result as illustrated in Figure 12 shows a more detailed base pressure distribution. This highlights one of the benefits of completing this study computationally. Further to this once slats are added to the base a numerical approach enables data collection not only for the near slat flow but also on the slat surfaces themselves. This would be difficult and time consuming to achieve experimentally due to the need to add tappings to all surfaces of interest which in some cases is simply impractical.

Analysis of instantaneous and time-averaged three-dimensional wake structures is also easily available within the computational data set; with considerable detail and small features being captured. It can be seen in Figure 15 that there is a high level of vortex shedding occurring from each of the trailing edges of the model; further indicating how well resolved the flow field is. This drives a lateral motion of the wake as described by Volpe et al. [7] due to the shedding from the model sides being out of phase. Close to the base the smallest vortical structures are observed and moving downstream the structures coalesce, increasing in size until they are shed from the free stagnation point. This shedding results in a varying wake length, as defined by Duell \& George [44] as wake pumping.

When averaged (Figure 16), these instantaneous vortices result in the wake toroid expected from the previous mid-plane analysis and as presented for the similar Ahmed geometry by Dalla Longa et al. [45] and Lucas et al. [11]. Here, the relationship between base pressure and the wake toroid is quite clear as the lower arm of the toroid corresponds to the low pressure region, which is also a region of high velocity downward flow. This relationship has been documented previously by Lucas et al. [11] who captured one of the asymmetric bi-stable states of an Ahmed body wake and showed a bias time-averaged toroid for which the dominating recirculation correlated to the region of lowest base pressure.

\section{Results \& Discussion}

The base slats were added systematically to the model at four heights $\left(\mathrm{S}_{\mathrm{z}}^{*}=0.375,0.5\right.$, $0.625 \& 0.75)$ and here the results for each configuration are presented and discussed. 


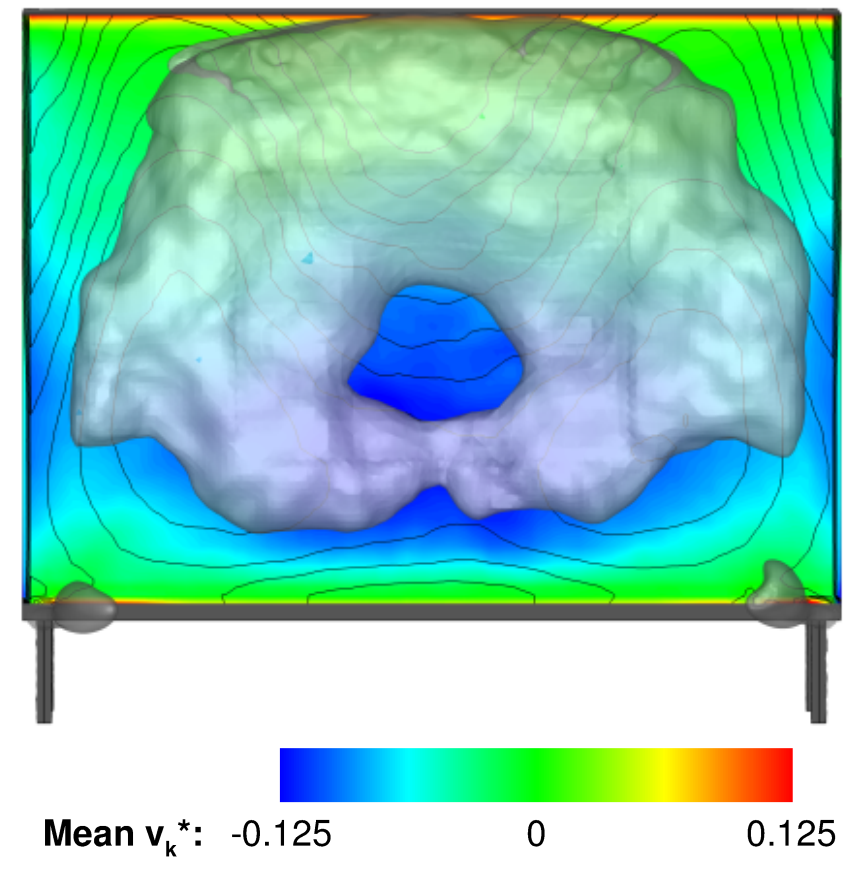

Figure 16: Base parallel plane, $0.5 \mathrm{~mm}$ downstream of the base, with vertical velocity as filled contours and pressure coefficient as contour lines along with a pressure isosurface $\left(C_{P}=-0.25\right)$ to visualise the time-averaged wake vortex ring for the baseline flow field.

For the lift and drag coefficients a statistical analysis was required to estimate the uncertainty in these values. As identified by Gaylard et al. [42] there is a level of dependence between any two subsequent samples taken from a force history due to the development of time-dependent motions within the wake which drive the forces experienced by the model. This means a force history cannot be considered as statistically independent samples making traditional analysis inapplicable. To resolve this issue Islam et al. outlined a method of determining a resampled data set to remove the statistical dependence in the unsteady signal which has been applied in this case [46]. Figure 17 shows the autocorrelation function of the time-dependent drag coefficient for the baseline configuration, with $95 \%$ significance limits. From this figure it can be seen that with a lag of approximately 0.035 seconds the significance of the autocorrelation is removed and so the data can be considered statistically independent.

Therefore the data was averaged over blocks of this size to give a new time series to be used for the statistical analysis. Then 95\% confidence intervals (CI) could be estimated using Equations 6 \& 7. 


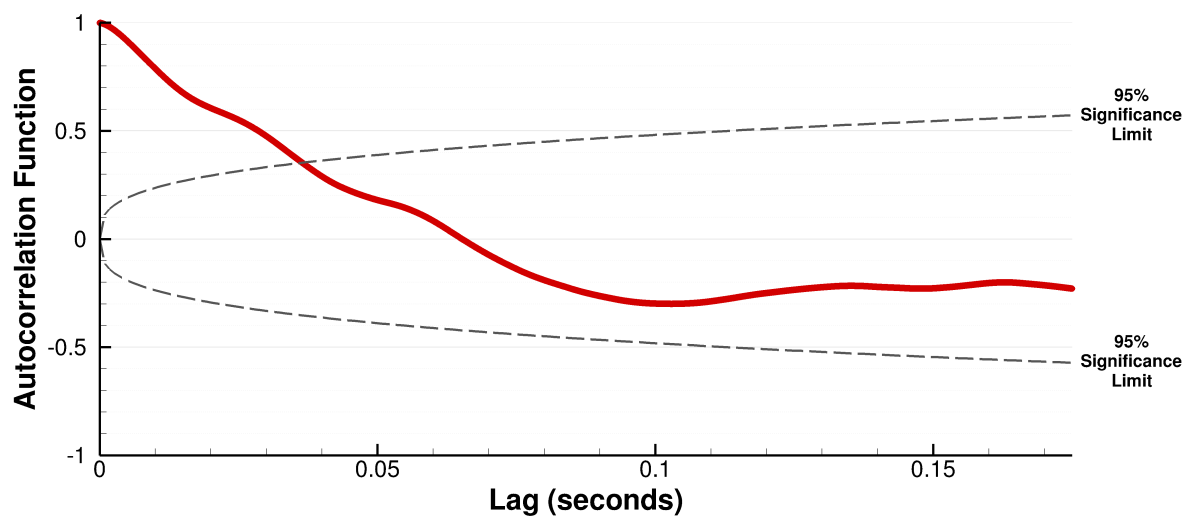

Figure 17: Autocorrelation function for the baseline configuration drag coefficient with $95 \%$ significance limits

$$
\begin{gathered}
e=t_{(0.05, n-1)} \frac{s}{\sqrt{n}} \\
C I=\bar{x} \pm e
\end{gathered}
$$

where the uncertainty error is denoted by e, the standard deviation $\mathrm{s}$, the mean $\bar{x}$ and the number of averaged blocks n.

This analysis was later applied to each slat configuration, enabling the uncertainty in the delta $\left(\mathrm{e}_{\Delta}\right)$ between the baseline and the slat configuration to be estimated using Equation 8.

$$
e_{\Delta}=\sqrt{\left(e_{b}\right)^{2}+\left(e_{\text {slat }}\right)^{2}}
$$

The changes in the lift and drag coefficients are summarised in Figure 18, with the statistical errors in the deltas illustrated via error bars. This shows significant reductions in drag for the two configurations with a slat above mid-base height and significant changes in lift for the lower $\left(\mathrm{S}_{\mathrm{z}}^{*}=0.325\right)$, mid-base $\left(\mathrm{S}_{\mathrm{z}}^{*}=0.5\right)$ and upper-quarter $\left(\mathrm{S}_{\mathrm{z}}^{*}=0.75\right)$ slats. Inspection of the surface pressures over the entire model indicated the changes in lift are local to the base region and are due to a combination of changes to the pressures on the diffuser surface and a pressure differential between the upper and lower slat surfaces.

The changes in drag are mainly due to changes in base pressure drag, with the skin friction drag remaining relatively unchanged due to the slat having a very small surface area and being placed in a region of separated flow. To further understand the changes in 


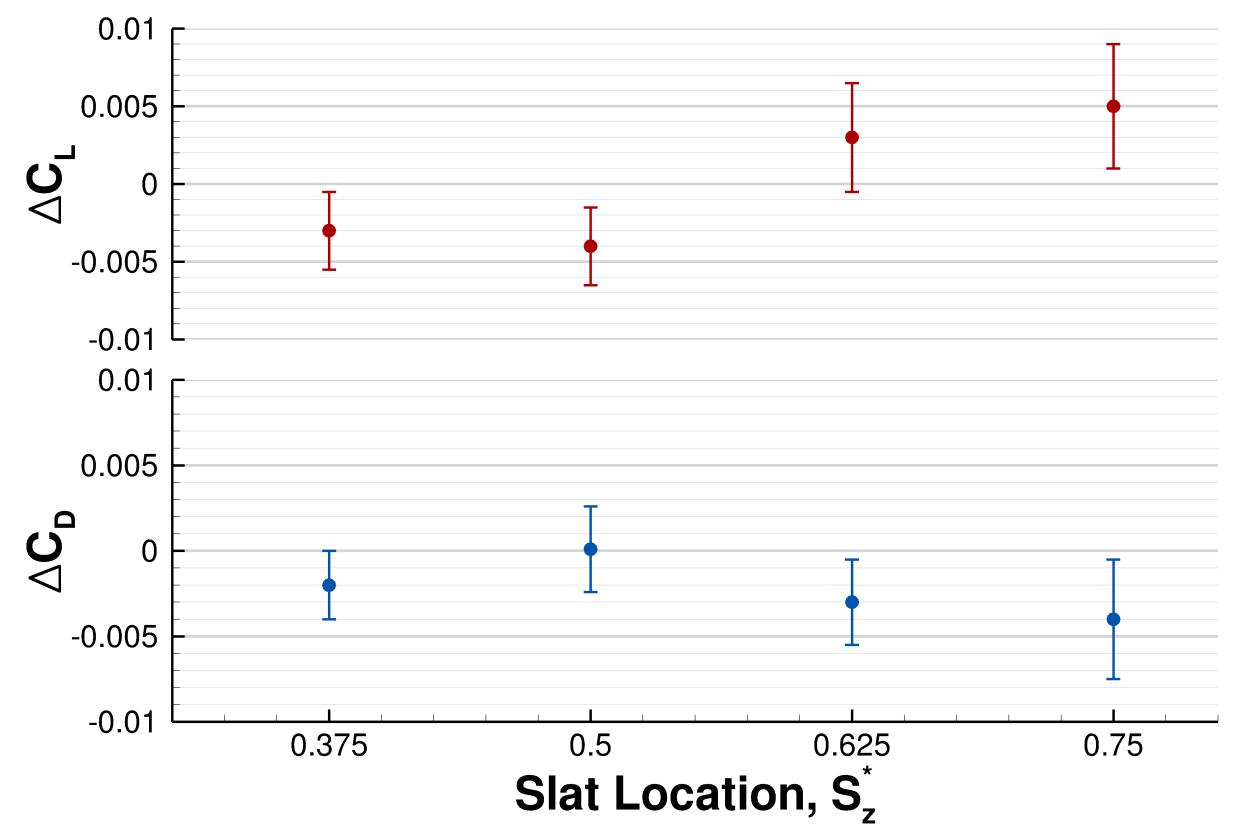

Figure 18: Changes in drag and lift coefficients compared to the baseline configuration for each tested slat location, with uncertainty due to fluctuations in the signal indicated via error bars.

base pressure drag, the contribution was calculated for the areas above and below the slat separately as illustrated in Figure 19. This shows that for the mid-base $\left(S_{z}^{*}=0.5\right)$ slat, the benefit of reduced drag above the slat is cancelled out by the increase in drag below the slat, explaining why the drag of the model was relatively unchanged. When the slat is moved down, to $\mathrm{S}_{\mathrm{z}}^{*}=0.375$, the gains above the slat are retained but the penalties below are reduced due to a smaller area of low pressure, enabling an overall drag reduction to be obtained. For the two slat configurations above mid-base height the base pressure drag both above and below the slat reduces due to an increase in pressure over the entire base surface. Therefore, explaining the overall, statistically significant, drag reductions of approximately 4 counts seen for these configurations.

The changes to the base pressure can be identified from the base pressure distributions in Figure 20. Localised changes are present in all cases, with an increase in pressure seen directly above each slat and a reduction seen directly below. For the lower $\left(\mathrm{S}_{\mathrm{z}}^{*}=0.325\right)$ and mid-base $\left(\mathrm{S}_{\mathrm{z}}^{*}=0.5\right)$ slats these local changes are largest in magnitude and so dominate the changes seen; leading to the split in behaviour above and below the slat for the base pressure drag, as identified previously. For the upper $\left(\mathrm{S}_{\mathrm{z}}^{*}=0.625\right)$ and upper-quarter $\left(\mathrm{S}_{\mathrm{z}}^{*}=0.75\right)$ slat configurations a more global change is seen instead, with increases in pressure seen over the 


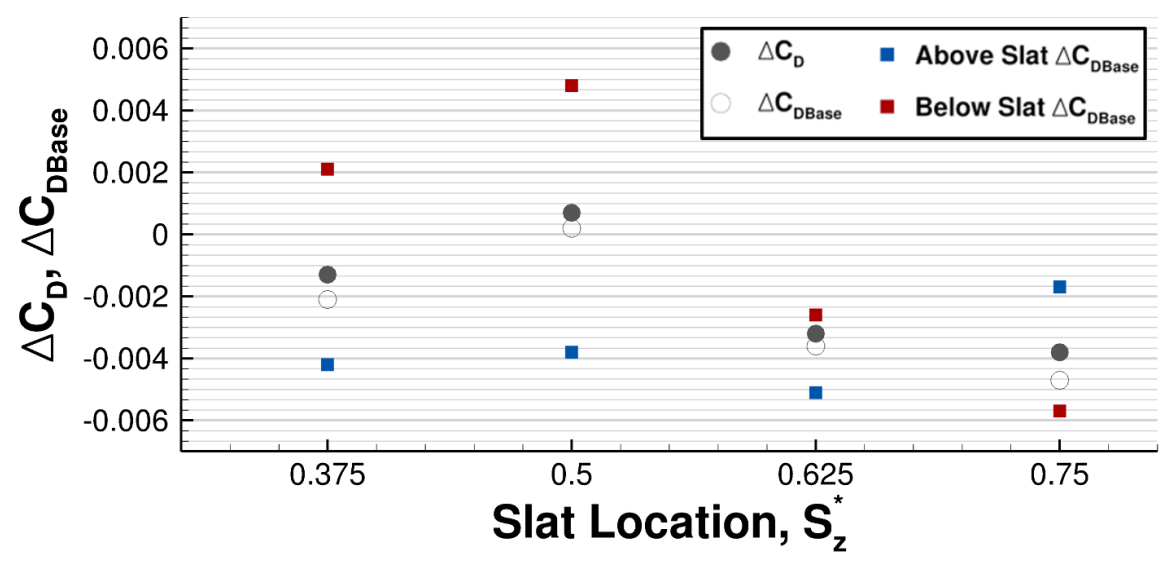

Figure 19: Changes in drag coefficient and base pressure drag coefficient.

entire base surface.

The mechanisms responsible for the localised near slat pressure changes are illustrated in Figure 21. Here the flow within the lower recirculation can be seen to approach the base surface before turning to travel parallel to the base. The vertical velocity of this flow increases as it approaches the slat until it stagnates on the upper slat surface. This stagnation is indicated by a red marker in Figure 21 and results in an increased pressure region around the centreline of the model. This higher pressure is observed not only on the stagnation surface but is also seen to spread to the neighbouring base surface. Figure 22 shows the pressure distribution over the upper surface of each slat and highlights that this impingement is present in each configuration; whilst also illustrating the distribution is almost independent of slat location. The upper $\left(\mathrm{S}_{\mathrm{z}}^{*}=0.625\right)$ and upper-quarter $\left(\mathrm{S}_{\mathrm{z}}^{*}=0.75\right)$ slats have a slightly higher pressure on the whole, and the lower $\left(\mathrm{S}_{\mathrm{z}}^{*}=0.325\right)$ and mid-base $\left(\mathrm{S}_{\mathrm{z}}^{*}=0.5\right)$ slats show some lower pressure regions toward the downstream edge of the slat as expected given they are placed in the low pressure region of the base pressure distribution.

The slat is also seen to create an obstacle to the recirculating flow with some streamlines being diverted downwards, rather than follow a straight path to the base. This combined with the impinging flow on the upper slat surface results in a region of separation directly below the slat. Within this separated region a vortex is formed, as visualised in Figure 21 by a blue streamtrace. This structure is seen to rotate the flow and drive it outward to be entrained in the free shear layers emanating from the model sides. The rotational strength of this structure is greatest at the centreline of the model and reduces as it extends to the model edges, with the width over which a high rotational velocity is seen being dependent 

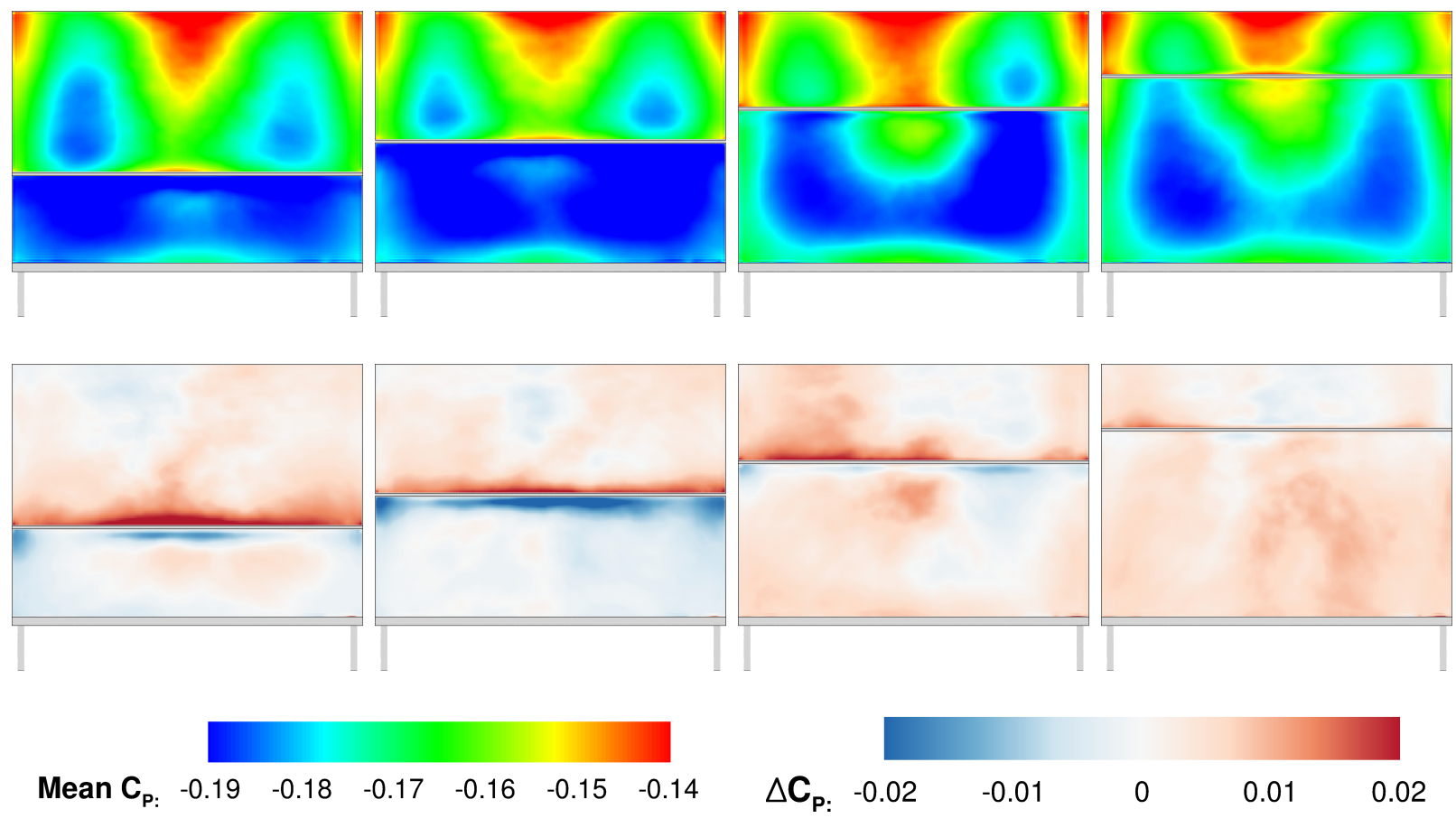

0.02

Figure 20: Above: Base pressure distributions.

Below: Changes in base pressure compared to the baseline case.

Left to Right: $\mathrm{S}_{\mathrm{z}}^{*}=0.375, \mathrm{~S}_{\mathrm{z}}^{*}=0.5, \mathrm{~S}_{\mathrm{z}}^{*}=0.625, \mathrm{~S}_{\mathrm{z}}^{*}=0.75$.

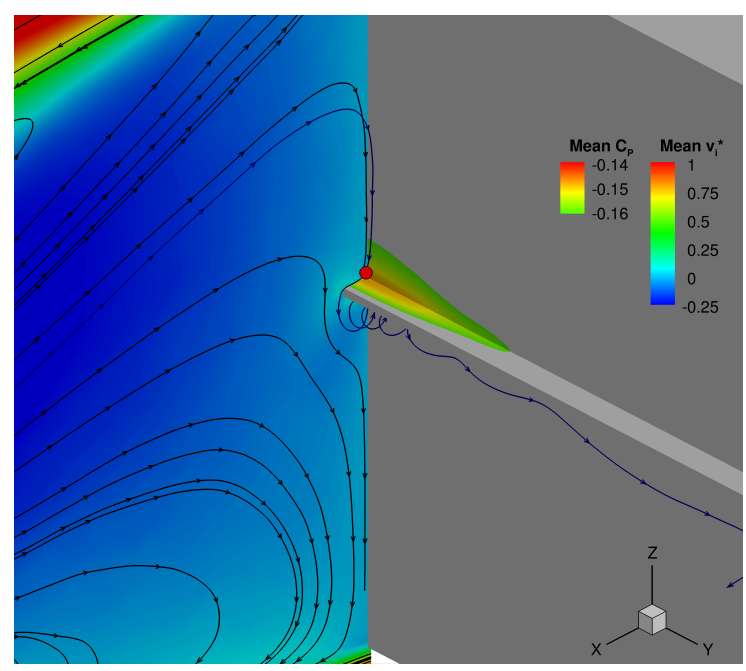

Figure 21: Local slat mechanisms illustrated on the mid-base slat configuration; upper slat surface impingement indicated by red marker \& below slat separation vortex visualised via blue streamtrace. 
Upper Surface of Slat $\left(S_{z}^{*}=0.375\right)$

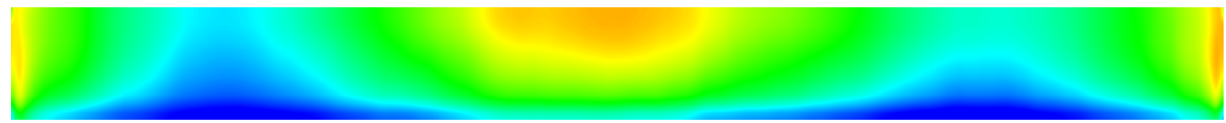

Upper Surface of Slat $\left(S_{z}^{*}=0.5\right)$

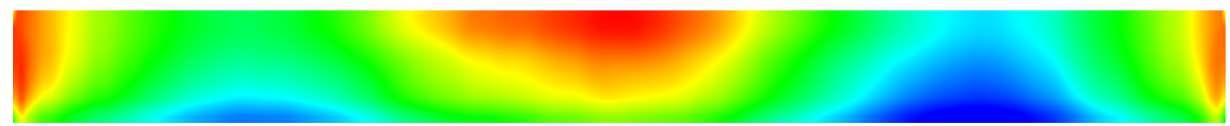

Upper Surface of Slat $\left(S_{z}^{*}=0.625\right)$

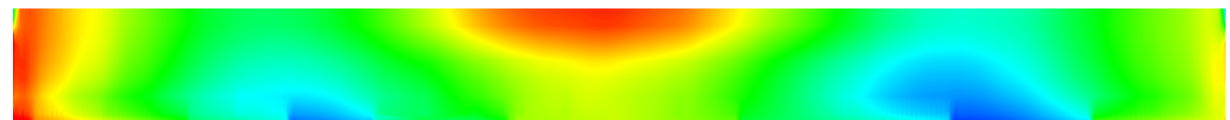

Upper Surface of Slat $\left(S_{z}^{*}=0.75\right)$

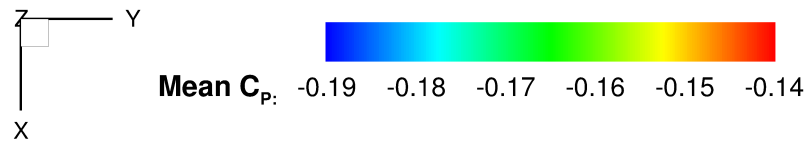

Figure 22: Pressure distribution over the upper surface of each tested slat.
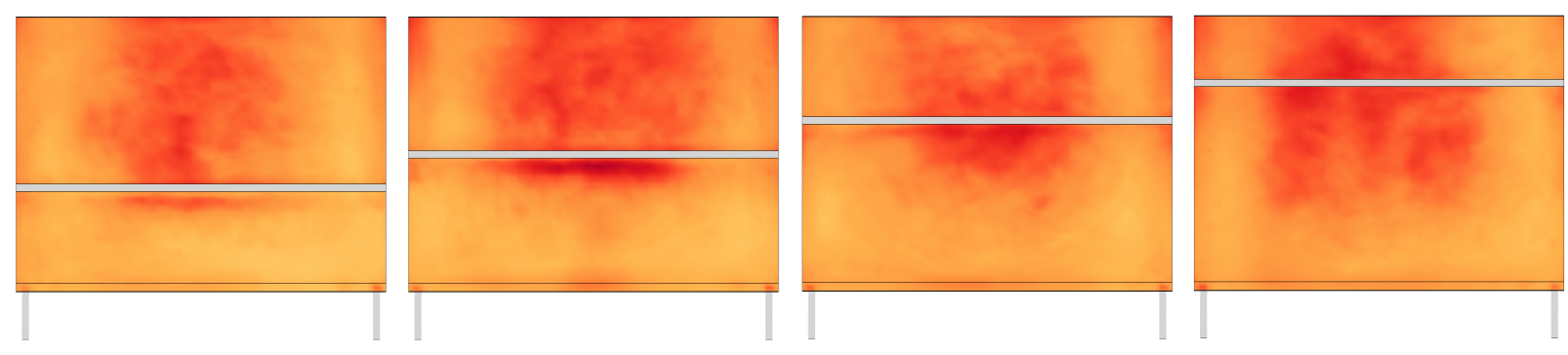

\section{$\begin{array}{llllll}\operatorname{RMS}\left(\mathbf{C}_{\mathrm{P}}{ }^{\prime}\right): & 0 & 0.01 & 0.02 & 0.03 & 0.04\end{array}$}

Figure 23: Root mean square of the pressure fluctuation on the base surface. Left to Right: $\mathrm{S}_{\mathrm{z}}^{*}=0.375, \mathrm{~S}_{\mathrm{z}}^{*}=0.5, \mathrm{~S}_{\mathrm{z}}^{*}=0.625, \mathrm{~S}_{\mathrm{z}}^{*}=0.75$. 
on slat location. For the mid-base $\left(\mathrm{S}_{\mathrm{z}}^{*}=0.5\right)$ slat configuration the rotational strength of the vortex is greater over a larger width than in any other case. This is due to the slat being a more substantial obstacle to the flow than at any other location; resulting in the largest separated region. This can be visualised by considering the RMS of the fluctuations in the base pressure coefficient as in Figure 23. Here for the mid-base slat $\left(S_{z}^{*}=0.5\right)$ a region of high RMS can be seen in the location of the vortex formed in the separated region below the slat. The highest values are seen over the central portion of the base and dissipate toward the model edges.

The magnitude of these localised pressure changes varies depending on the slat location as illustrated in Figure 24, which shows the pressure coefficient on the base surface along the line $y=0$. All the slat locations show very similar pressure profiles, which away from the slat converge close to the baseline profile. For the cases with a more global pressure increase, such as the upper-quarter $\left(\mathrm{S}_{\mathrm{z}}^{*}=0.75\right)$ slat, the same profile shape is observed but translated to higher pressure values. Additionally the localised changes are clearly demonstrated by the sharp deviations in pressure directly above and below each slat location, acting over a similar vertical distance in each case. The magnitudes of change differ, with the mid-base $\left(\mathrm{S}_{\mathrm{z}}^{*}=0.5\right)$ slat showing the largest, and the upper-quarter $\left(\mathrm{S}_{\mathrm{z}}^{*}=0.75\right)$ slat the smallest, deviations. The spanwise effect of these near slat mechanisms is also dependent on slat location, as illustrated in Figure 25 which shows the base pressure coefficient along the lines $\mathrm{y}=-\mathrm{W} / 4$ and $\mathrm{y}=\mathrm{W} / 4$. It demonstrates that the mid-base $\left(S_{z}^{*}=0.5\right)$ slat has the largest off-centre effect with large pressure changes present away from the centreline. For the baseline configuration a high level of left to right symmetry is observed with the two off-centre pressure profiles being almost identical. However, with the addition of a slat the pressure profiles show larger differences between each side of the base, indicating an increased level of asymmetry in the wake. This is unexpected, given the model itself maintains horizontal symmetry. The vortex below the slat has been seen to feed into the free shear layers at the model edges which likely drives additional wake flapping and this may be the cause of the asymmetry in the time-averaged base pressure distribution. Across Figures $24 \& 25$ a clear similarity between pressure profiles for each slat configuration is observed, this further highlights the localised nature of the changes and indicates minimal impact on the more dominant wake features. At the higher slat locations the base pressure is higher than is seen where the lower $\left(\mathrm{S}_{\mathrm{z}}^{*}=0.325\right)$ and mid-base $\left(\mathrm{S}_{\mathrm{z}}^{*}=0.5\right)$ slats will be placed, therefore the deviation is due to the relative difference between this baseline base pressure and the impingement pressure, which is greatest in the mid-base $\left(\mathrm{S}_{\mathrm{z}}^{*}=0.5\right)$ slat configuration. 


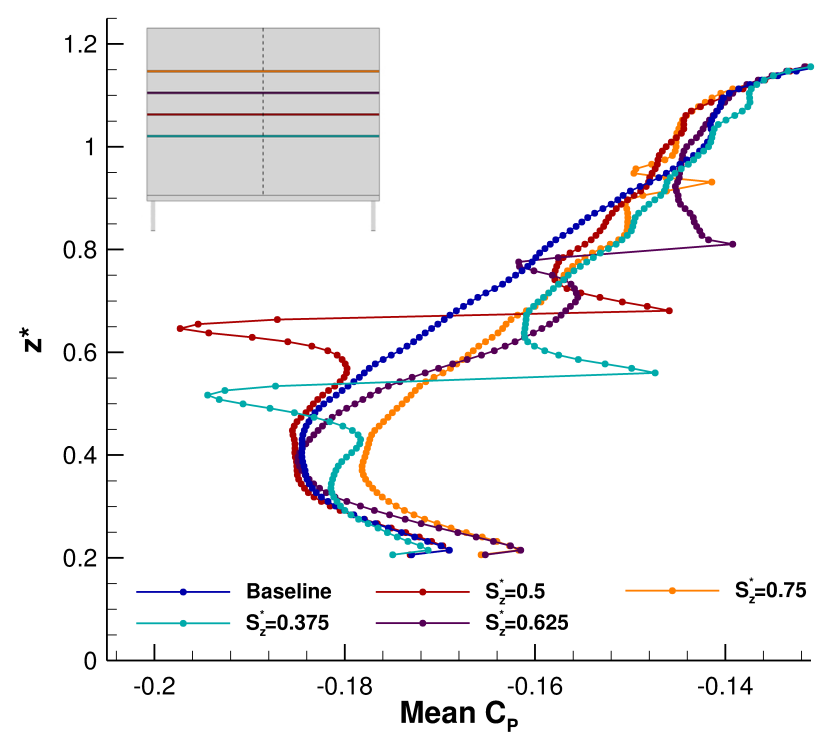

Figure 24: Pressure coefficient on the model base for each configuration along the line $\mathrm{y}=0$

The vortex located below the slat and identified in Figure 21 is present for each slat location (Figure 27). The magnitude of the low pressure attributed to this structure varies considerably due to the flow velocity and direction as it interacts with the slat. The near slat flow is shown for each slat configuration in Figure 27 where the different direction of the approaching flow can be clearly seen. The vortex is strongest in the mid-base $\left(\mathrm{S}_{\mathrm{z}}^{*}=0.5\right)$ slat configuration as the flow approaching the slat is travelling parallel to the base surface and so normal to the slat. This makes the slat a large obstacle to the flow and so the largest separated region is formed. The strength and size of the vortex can be visualised by considering vertical velocity as in Figure 28. Here it can be seen that the velocity both above and below the slat are largest for the mid-base $\left(\mathrm{S}_{\mathrm{z}}^{*}=0.5\right)$ slat, as is the width of the high velocity region. This vortex is shown to have similar vorticity to the structures within the free shear layers (Figure 26), further indicating the significance of the structure. The lower $\left(\mathrm{S}_{\mathrm{z}}^{*}=0.325\right)$ slat is placed closer to the lower recirculation and so the flow direction is changed, as the flow now approaches the base at an angle the slat impedes less of the flow; resulting in a smaller separation and weaker vortex. The same can be said for the upper $\left(\mathrm{S}_{\mathrm{z}}^{*}=0.625\right)$ and upper-quarter $\left(\mathrm{S}_{\mathrm{z}}^{*}=0.75\right)$ slats. In these cases the flow has yet to be turned fully as it approaches the slat and so again has an easier path to the base, resulting in an even weaker separation vortex.

Furthermore, the vertical velocity attributed to the lower recirculation is reduced in magnitude corresponding to increased pressure. This explains the reduction in drag below 

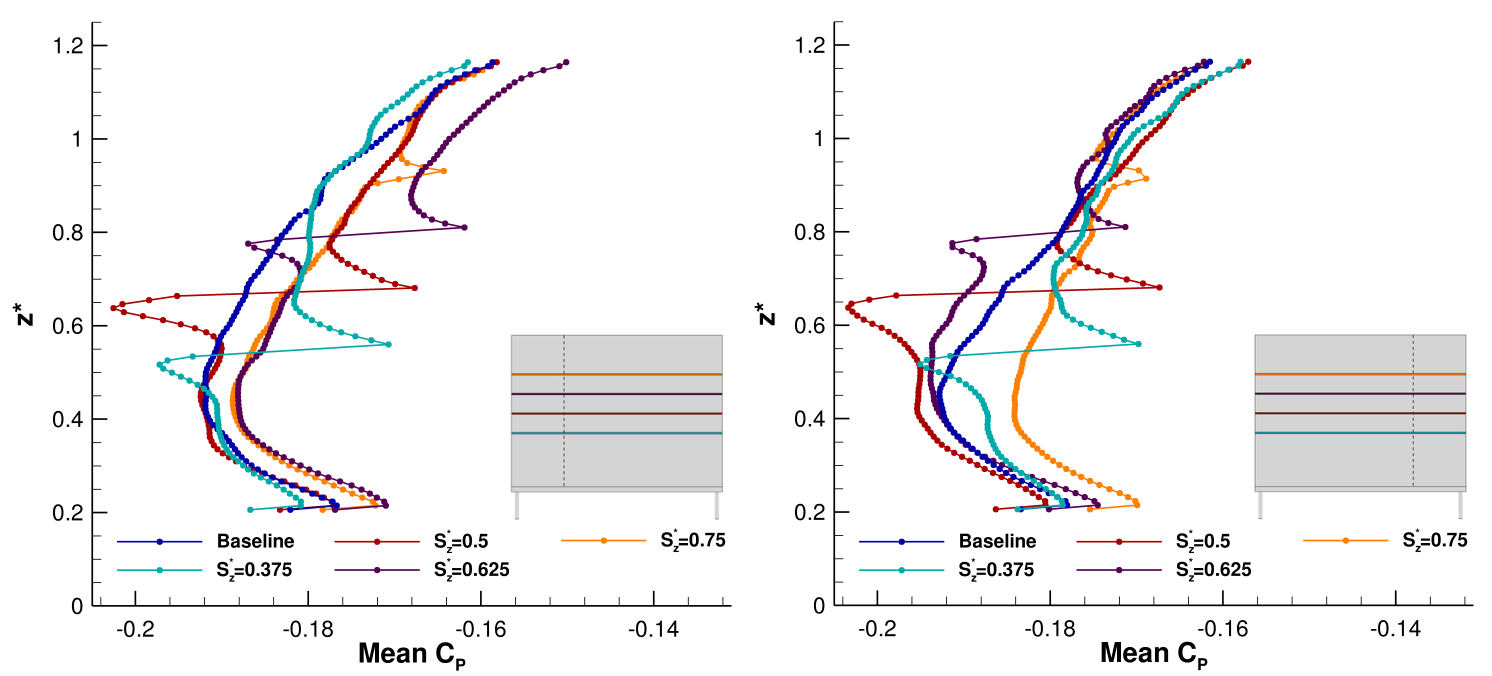

Figure 25: Pressure coefficient on the model base for each configuration along the lines $\mathrm{y}=-\mathrm{W} / 4$ (left) \& $\mathrm{y}=\mathrm{W} / 4$ (right).

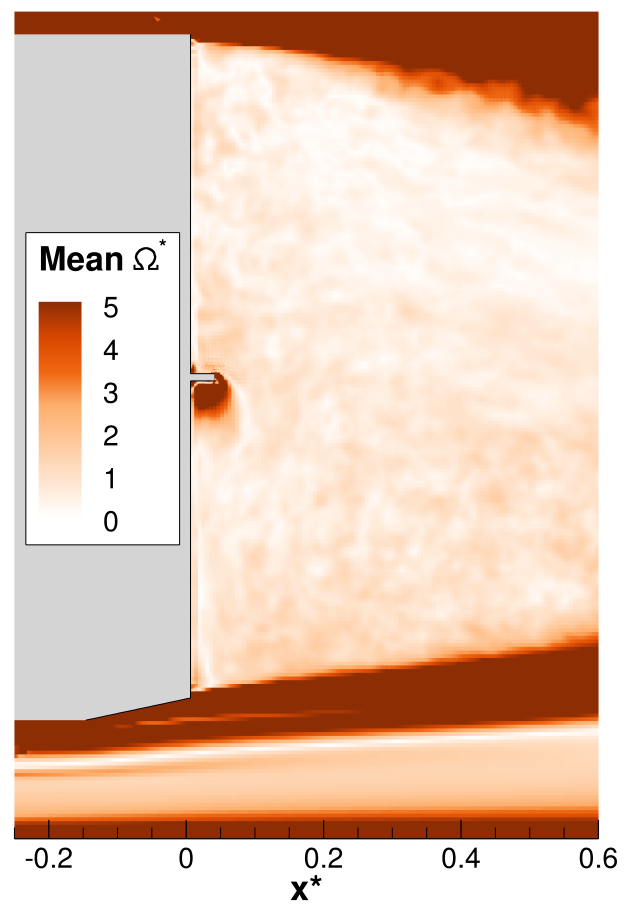

Figure 26: Normalised vorticity magnitude in the $\mathrm{y}=0$ mid-plane of the wake for the mid-base $\left(\mathrm{S}_{\mathrm{z}}^{*}=0.5\right)$ slat configuration. 


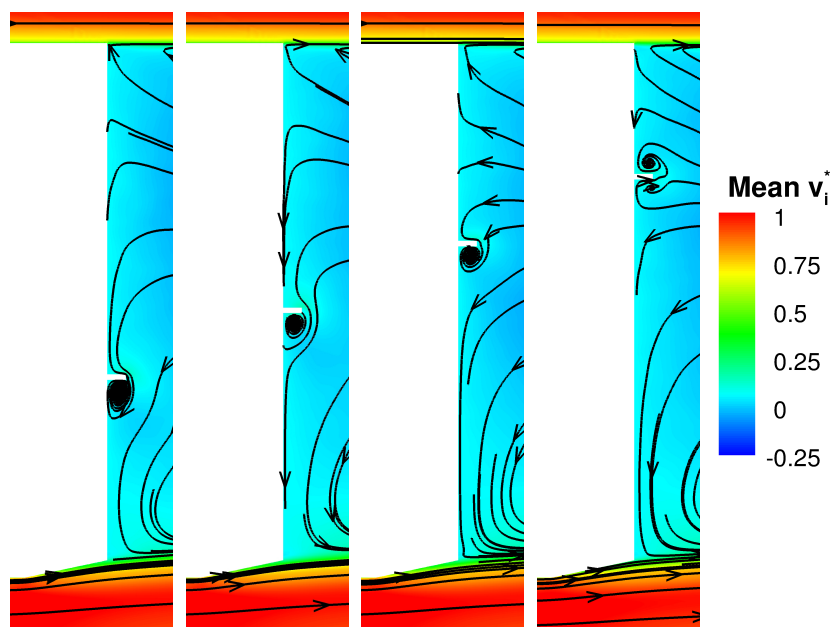

Figure 27: Vertical mid-plane $(\mathrm{y}=0)$ near the base surface.

Left to Right: $\mathrm{S}_{\mathrm{z}}^{*}=0.375, \mathrm{~S}_{\mathrm{z}}^{*}=0.5, \mathrm{~S}_{\mathrm{z}}^{*}=0.625, \mathrm{~S}_{\mathrm{z}}^{*}=0.75$.
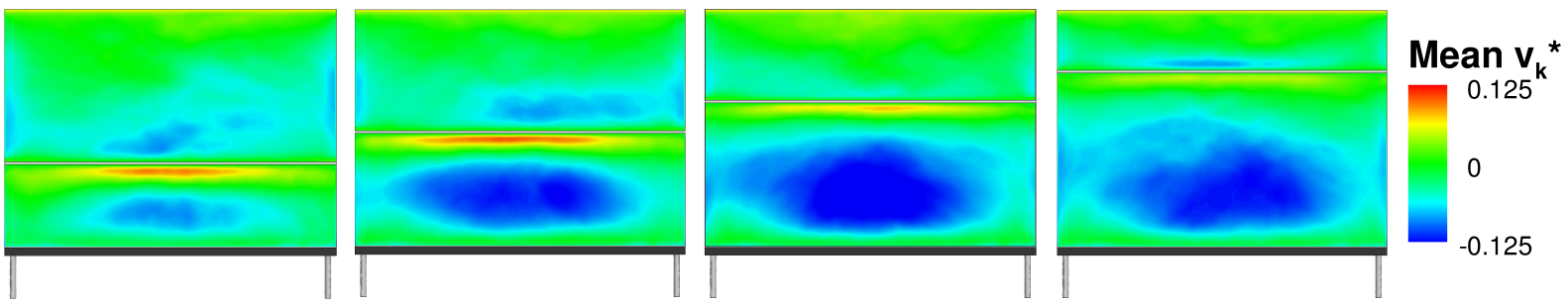

Figure 28: Vertical velocity in a base parallel plane, $0.5 \mathrm{~mm}$ downstream of the base, for each slat configuration.

Left to Right: $\mathrm{S}_{\mathrm{z}}^{*}=0.375, \mathrm{~S}_{\mathrm{z}}^{*}=0.5, \mathrm{~S}_{\mathrm{z}}^{*}=0.625, \mathrm{~S}_{\mathrm{z}}^{*}=0.75$.

the slat for the upper $\left(\mathrm{S}_{\mathrm{z}}^{*}=0.625\right)$ and upper-quarter $\left(\mathrm{S}_{\mathrm{z}}^{*}=0.75\right)$ slats as this pressure increase dominates over the weaker separation vortices. The effect is still present in the other two configurations, however the low pressure associated with the vortex located directly below the slat dominates in these cases, leading to an increase in drag for the entire area below the slat. This implies the near slat mechanisms are disrupting the lower recirculating structure enough to slow its rotational velocity, however the significance of the pressure increase due to this is dependent on the magnitude of the low pressure vortex formed in the separated region below the slat.

For the upper-quarter $\left(\mathrm{S}_{\mathrm{z}}^{*}=0.75\right)$ slat a neater region of higher velocity downward flow is seen above the slat, on inspection this is due to the formation of a vortex above the slat as well as below; as highlighted in Figure 27. Only the near base field has been included as the main recirculating structures are unaffected by the addition of a slat at any height. It is worth noting that this is also true for those seen in the horizontal mid-plane. 
Base Surface of Slat $\left(S_{z}^{*}=0.375\right)$

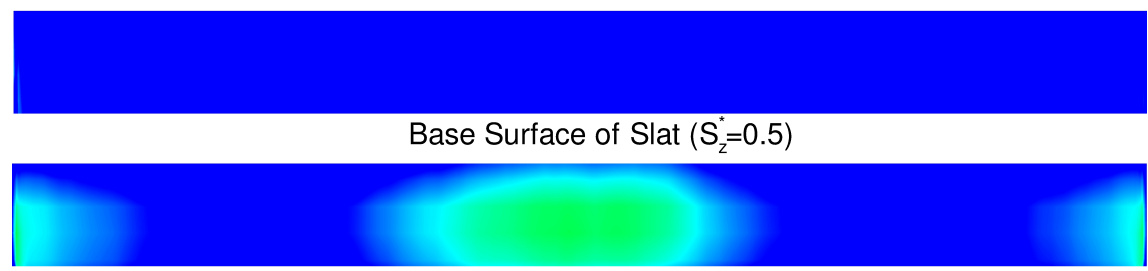

Base Surface of Slat $\left(\mathrm{S}_{\mathrm{z}}^{*}=0.625\right)$

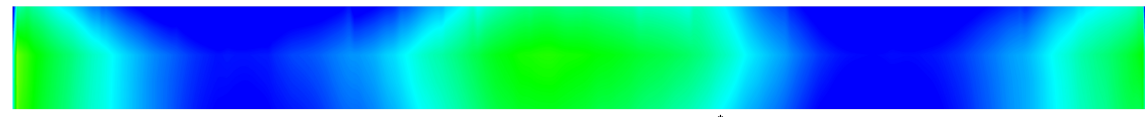

Base Surface of Slat $\left(\mathrm{S}_{\mathrm{z}}^{*}=0.75\right)$

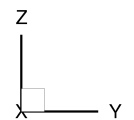

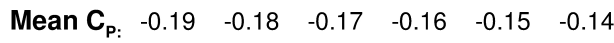

Figure 29: Pressure distribution over the base parallel surface of each tested slat.

This indicates the slat's proximity to the base impingement is important with regards to the near slat mechanisms, however there are further benefits available by placing the slat close to the impingement. As can be seen in Figure 20 for both the upper $\left(\mathrm{S}_{\mathrm{z}}^{*}=0.625\right)$ and upper-quarter $\left(\mathrm{S}_{\mathrm{z}}^{*}=0.75\right)$ slats there is a region of high pressure lower on the base surface than in the baseline configuration. This is due to the slat increasing the area over which the flow approaching the base stagnates, resulting in multiple impingement locations. One of these being below the slat, whilst there remains an impingement above the slat, along with a further impingement on the base surface of the slat as indicated by the higher pressure region around $\mathrm{y}=0$ in Figure 29. The increased region of base impingement is also evidenced in the RMS values shown in Figure 23, whereby for the upper $\left(\mathrm{S}_{\mathrm{z}}^{*}=0.625\right)$ and upper-quarter $\left(\mathrm{S}_{\mathrm{z}}^{*}=0.75\right)$ slat configurations the region of high RMS is seen to extend further toward the lower edge of the model.

The bulk mean wake structures have not been radically changed with the addition of a base slat. Although only illustrated here for two cases, the lower $\left(\mathrm{S}_{\mathrm{z}}^{*}=0.325\right)$ and upper $\left(S_{z}^{*}=0.625\right)$ slats (which are deemed to be representative of all cases), Figure 30 shows the time-averaged wake toroid is still present. The pressure iso-surfaces are less complete with a base slat than was seen for the baseline configuration in Figure 16. This indicates increased unsteadiness within the flow, which is also seen when considering the model side force. Assuming, for this model, a symmetric mean flow field would be expected the RMS of side 
Table 3: RMS of side force for each slat configuration, normalised by the baseline value

\begin{tabular}{|l|c|}
\hline & RMS Side Force \\
\hline Baseline & 1 \\
Lower-Slat & 1.20 \\
Mid-Base Slat & 1.42 \\
Upper-Slat & 1.59 \\
Upper-Quarter Slat & 1.37 \\
\hline
\end{tabular}

force was considered to determine the unsteadiness and instantaneous asymmetry within the wake. To aid the comparison between baseline and slat configurations the RMS of side force has been normalised by the baseline value as presented in Table 3. From this it can be seen that there is an increase for every slat configuration, providing further evidence of the slat's ability to increase the unsteadiness and instantaneous asymmetry within the wake. This is the highest for the upper-quarter $\left(\mathrm{S}_{\mathrm{z}}^{*}=0.75\right)$ slat configuration, with the fluctuations increasing by almost $60 \%$. The vortical structure formed in the separated region below the slat has been seen in Figure 21 to feed into the free shear layers at the model sides, this interaction drives an increased flapping of the main wake structures resulting in the higher level of instantaneous asymmetry. Despite these instantaneous modifications to the bulk flow structures, once the field is averaged the effect is minimal as the averaging window is sufficient to capture a balance of both left and right biased asymmetric states.

Further minor modifications to the bulk flow have been observed when the velocities of the main recirculating structures are considered. Figure 30 shows a reduction in the streamwise velocity of the under-body flow entrained into the lower recirculation for the upper $\left(\mathrm{S}_{\mathrm{z}}^{*}=0.625\right)$ slat configuration. This effect is also observed for the upper-quarter $\left(\mathrm{S}_{\mathrm{z}}^{*}=0.75\right)$ slat and is seen to increase the effectiveness of the base impingement as the velocity of flow stagnating on the base surface below the slat is increased; resulting in an additional region of higher pressure. When the lower $\left(\mathrm{S}_{\mathrm{z}}^{*}=0.325\right)$ and mid-base $\left(\mathrm{S}_{\mathrm{z}}^{*}=0.5\right)$ slats are considered a different mechanism is seen. Rather than alterations to the impingement of the lower recirculation, the vertical velocity of the near wall flow is seen to be reduced (Figure 30). This indicates, as hypothesised by Littlewood [21], a reduction in rotational energy of the lower arm of the wake toroid resulting in an increase in the surface pressure corresponding to this structure.

These effects highlight how changes local to the slats result in subtle modifications to the more dominant flow structures. There is a significant cumulative effect when all the observed changes are considered despite the bulk mean flow features being maintained. This highlights 

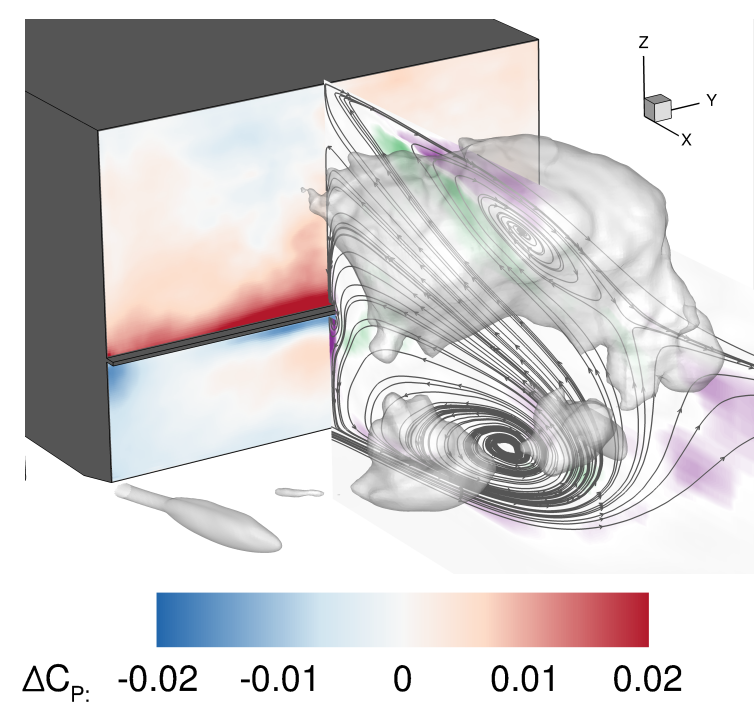

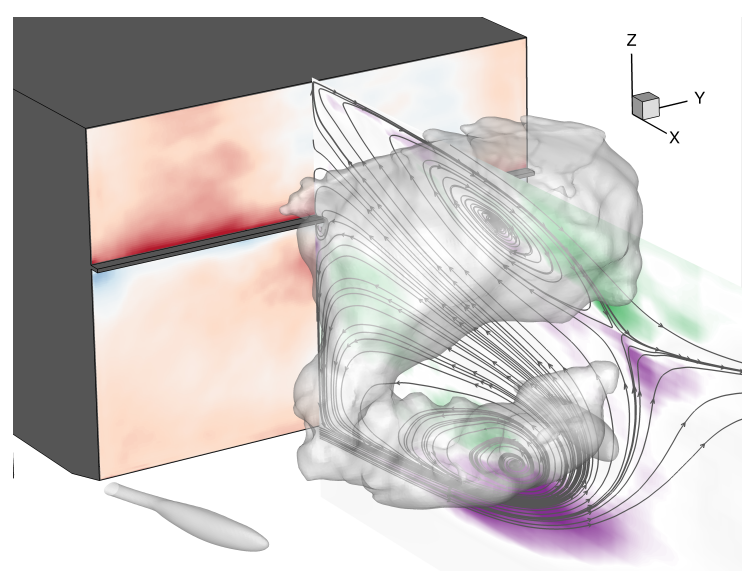

$\Delta \mathrm{v}^{*}:-0.1$

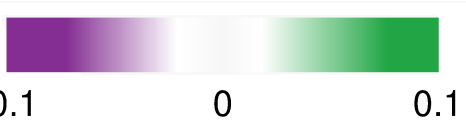

Figure 30: Changes in base pressure coefficient and planar velocity in $\mathrm{y}=0$ mid-plane compared to the baseline for lower-slat (left) \& upper-slat (right) with a pressure isosurface $\left(C_{P}=-0.25\right)$ illustrating the time-averaged vortex ring.

the need for high resolution analysis, in particular, near model surfaces when analysing the effects of geometry changes.

\section{Conclusions}

A well validated CFD methodology has been successfully implemented to isolate and identify the mechanisms responsible for flow changes seen when applying small base geometry modifications to a simplified vehicle.

The baseline flow field shows time-dependent vortical structures are shed from each of the trailing edges of the model, forming a highly unsteady and three-dimensional wake. These structures result in a vertically asymmetric time-averaged vortex ring, with the asymmetry attributed to the lower taper increasing the upwash within the wake. These time-averaged wake structures remain relatively unchanged with the addition of a base slat. Despite this, the addition of a base slat causes statistically significant changes to both lift and drag. The best case, a slat placed at $\mathrm{S}_{\mathrm{z}}^{*}=0.75$, resulted in a drag reduction of approximately 4 counts which is equivalent to a saving of $0.5 \mathrm{gCO}_{2} / \mathrm{km}$ or an increase in electric vehicle range of 2 $\mathrm{km}$.

Drag reductions are shown to be due to an increase in pressure over the entire base surface caused by changes to the near-wall vertical velocity associated with the lower recirculation and changes to the base impingement, including promoting multiple impingement zones. The 
slat also reduces the velocity of the under-body flow entering the wake, resulting in increased impingement velocity and further increasing the benefit of these multiple impingements. The cumulative result of all these increased pressure regions is a significant reduction in base pressure drag and so overall model drag.

For all slat locations tested local effects result in pressure changes in the regions directly above and below the slats. A high-pressure region is seen directly above each slat and is due to recirculating flow impinging on the upper slat surface. The magnitude of this pressure increase is similar for all slats considered. Below each slat a region of separation induces a vortical structure that becomes entrained in the free shear layers at the slat ends. This drives additional flapping of the wake, increasing the instantaneous asymmetry. The strength of the separation vortex is dependent on the local approach flow and hence on the slat location and as the vortex strength reduces there is a smaller reduction in pressure. The results highlight how small geometry change can influence the body forces, whilst having minimal effect on dominating wake structures. This demonstrates that the conventional approach that relies on controlling separation and increasing pressure recovery should be supplemented with efforts to control the interaction between recirculating flow and the base surface.

A limited number of configurations have been explored here so combinations of slats may enable further drag reduction to be achieved. For example by combining an upper slat with a modified lower slat designed to prevent separation on the lower surface. Such an approach may allow the gains in global pressure to be supplemented with the localised benefits seen on the upper surface of the lower slat.

\section{Acknowledgements}

The author would like to thank Giancarlo Pavia for providing additional experimental data. This project is partially funded by Jaguar Land Rover.

\section{Nomenclature}

$S_{z}^{*}$ - Normalised Slat Location

$C_{P}$ - Surface Pressure Coefficient

$p$ - Recorded Surface Pressure

$p_{\infty}$ - Free-Stream Static Pressure

$\rho$ - Air Density 
F - Measured Model Force

$u_{\text {corrected }}$ - Corrected Free Stream Velocity

$C_{D}$ - Drag Coefficient

$C_{\text {Dbase }}$ - Base Pressure Drag Coefficient

$A$ - Base Surface Area

$A_{i}$ - Projected Associated Area for a Given Tap
$647 s$ - Standard Deviation for Block Averaged Time

648 Series

$649 \bar{x}$ - Mean for Block Averaged Time Series

$650 \quad n$ - Number of Blocks

${ }_{651} e_{\Delta}$ - Uncertainty Error in Force Coefficient Delta

$652 e_{b}$ - Uncertainty Error in Baseline Configuration

$653 e_{\text {slat }}$ - Uncertainty Error in Slat Configuration

\section{References}

[1] M. Martin, A. Eichberger, E. Dragoti-Cela, Optimization approach to handle global co2 fleet emission standards, in: SAE Technical Paper 2016-01-0904, SAE International, 2016. doi:10.4271/2016-01-0904. URL https://doi.org/10.4271/2016-01-0904

[2] W. S. Duff, R. Dowling, B. Hung, G. Lancaster, L. Ridge, Simulation of auto design performance in the market to meet fuel efficiency standards, in: 2015 Winter Simulation Conference (WSC), 2015, pp. 3160-3161. doi:10.1109/WSC.2015.7408449.

[3] A. Wood, M. Passmore, D. Forbes, D. Wood, A. Gaylard, Base pressure and flow-field measurements on a generic suv model, SAE Int. J. Passeng. Cars - Mech. Syst. 8 (2015) 233-241. doi:10.4271/201501-1546.

URL https://doi.org/10.4271/2015-01-1546

[4] A.-K. Perry, M. Almond, M. Passmore, R. Littlewood, The study of a bi-stable wake region of a generic squareback vehicle using tomographic piv, SAE Int. J. Passeng. Cars - Mech. Syst. 9 (2016) 743-753. doi:10.4271/2016-01-1610. URL https://doi.org/10.4271/2016-01-1610

[5] B. Khalighi, S. Zhang, C. Koromilas, S. Balkanyi, L. P. Bernal, G. Iaccarino, P. Moin, Experimental and computational study of unsteady wake flow behind a bluff body with a drag reduction device, in: SAE Technical Paper 2001-01-1042, SAE International, 2001. doi:https://doi.org/10.4271/2001-01-1042. URL https://doi.org/10.4271/2001-01-1042

[6] G. Pavia, M. Passmore, C. Sardu, Evolution of the bi-stable wake of a square-back automotive shape, Experiments in Fluids 59 (1) (2018) 20.

[7] R. Volpe, P. Devinant, A. Kourta, Experimental characterization of the unsteady natural wake of the full-scale square back ahmed body: flow bi-stability and spectral analysis, Experiments in Fluids 56 (5) (2015) 99. doi:10.1007/s00348-015-1972-0. URL https://doi.org/10.1007/s00348-015-1972-0

[8] M. Grandemange, M. Gohlke, O. Cadot, Turbulent wake past a three-dimensional blunt body. part 1. global modes and bi-stability, Journal of Fluid Mechanics 722 (2013) 51-84.

[9] B. Khalighi, K.-H. Chen, G. Iaccarino, Unsteady aerodynamic flow investigation around a simplified square-back road vehicle with drag reduction devices, Journal of Fluids Engineering 134 (6) (2012) 061101.

[10] Y. Irving Brown, S. Windsor, A. Gaylard, The effect of base bleed and rear cavities on the drag of an suv, 
in: SAE Technical Paper 2010-01-0512, SAE International, 2010. doi:https://doi.org/10.4271/2010-010512.

URL https://doi.org/10.4271/2010-01-0512

[11] J.-M. Lucas, O. Cadot, V. Herbert, S. Parpais, J. Délery, A numerical investigation of the asymmetric wake mode of a squareback ahmed body-effect of a base cavity, Journal of Fluid Mechanics 831 (2017) 675-697.

[12] P. Gilliéron, A. Kourta, Aerodynamic drag reduction by vertical splitter plates, Experiments in Fluids 48 (1) (2010) 1-16.

[13] L. Sterken, L. Lofdahl, S. Sebben, T. Walker, Effect of rear-end extensions on the aerodynamic forces of an suv, in: SAE Technical Paper 2014-01-0602, SAE International, 2014. doi:https://doi.org/10.4271/2014-01-0602.

URL https://doi.org/10.4271/2014-01-0602

[14] A.-K. Perry, M. Passmore, A. Finney, Influence of short rear end tapers on the base pressure of a simplified vehicle., SAE Int. J. Passeng. Cars - Mech. Syst. 8 (1) (2015) 317-327. doi:https://doi.org/10.4271/2015-01-1560.

URL https://doi.org/10.4271/2015-01-1560

[15] G. Pavia, M. Passmore, A. Gaylard, Influence of short rear end tapers on the unsteady base pressure of a simplified ground vehicle, in: SAE Technical Paper 2016-01-1590, SAE International, 2016. doi:https://doi.org/10.4271/2016-01-1590.

URL https://doi.org/10.4271/2016-01-1590

[16] J. Howell, M. Passmore, S. Tuplin, Aerodynamic drag reduction on a simple car-like shape with rear upper body taper, SAE Int. J. Passeng. Cars - Mech. Syst. 6 (1) (2013) 52-60. doi:https://doi.org/10.4271/2013-01-0462. URL https://doi.org/10.4271/2013-01-0462

[17] R. Verzicco, M. Fatica, G. Iaccarino, P. Moin, B. Khalighi, Large eddy simulation of a road vehicle with drag-reduction devices, AIAA journal 40 (12) (2002) 2447-2455.

[18] J. Howell, A. Sheppard, A. Blakemore, Aerodynamic drag reduction for a simple bluff body using base bleed, in: SAE Technical Paper 2003-01-0995, SAE International, 2003. doi:https://doi.org/10.4271/2003-01-0995.

URL https://doi.org/10.4271/2003-01-0995

[19] R. Littlewood, M. A. Passmore, Aerodynamic drag reduction of a simplified squareback vehicle using steady blowing, Experiments in fluids 53 (2) (2012) 519-529.

[20] D. Barros, J. Borée, B. R. Noack, A. Spohn, T. Ruiz, Bluff body drag manipulation using pulsed jets and coanda effect, Journal of Fluid Mechanics 805 (2016) 422-459.

[21] R. Littlewood, M. Passmore, D. Wood, An investigation into the wake structure of square back vehicles and the effect of structure modification on resultant vehicle forces, SAE International Journal of Engines 4 (2) (2011) 2629-2637. doi:https://doi.org/10.4271/2011-37-0015.

URL https://doi.org/10.4271/2011-37-0015

[22] S. Windsor, The effect of rear end shape on road vehicle aerodynamic drag, in: Autotech 1991. Seminar 6. Aerodynamics Update. Congress Seminar Papers.(Paper NO. C427/6/031), 1991.

[23] S. Ahmed, G. Ramm, G. Faltin, Some salient features of the time-averaged ground vehicle wake, Tech. 
rep., SAE Technical Paper (1984).

[24] I. Robertson, A. Becot, A. Gaylard, B. Thornber, Automotive drag reduction through distributed base roughness elements, Advances in Computational Mechanics 553 (2014) 267-272. doi:10.4028/www.scientific.net/AMM.553.267.

[25] D. Forbes, G. Page, M. Passmore, A. Gaylard, Computational study of wake structure and base pressure on a generic suv model, in: IMechE International Vehicle Aerodynamics Conference, 2014, pp. 67-77.

[26] A.-K. Perry, G. Pavia, M. Passmore, Influence of short rear end tapers on the wake of a simplified square-back vehicle: wake topology and rear drag, Experiments in Fluids 57 (11) (2016) 169.

[27] O. Ehirim, K. Knowles, A. Saddington, A review of ground-effect diffuser aerodynamics, Journal of Fluids Engineering 141 (2) (2019) 020801.

[28] A. Kabanovs, M. Varney, A. Garmory, M. Passmore, A. Gaylard, Experimental and computational study of vehicle surface contamination on a generic bluff body, in: SAE 2016 World Congress and Exhibition, SAE International, 2016. doi:https://doi.org/10.4271/2016-01-1604.

URL https://doi.org/10.4271/2016-01-1604

[29] P. Spalart, W.-H. Jou, M. Strelets, S. Allmaras, Comments on the feasibility of les for wings, and on a hybrid rans/les approach, in: Proceedings of first AFOSR international conference on DNS/LES, Greyden Press, 1997.

[30] T. Favre, B. Diedrichs, G. Efraimsson, Detached-eddy simulations applied to unsteady crosswind aerodynamics of ground vehicles, in: Progress in Hybrid RANS-LES Modelling, Springer, 2010, pp. 167-177.

[31] N. Ashton, A. Revell, Comparison of rans and des methods for the drivaer automotive body, in: SAE Technical Paper 2015-01-1538, SAE International, 2015. doi:https://doi.org/10.4271/2015-01-1538. URL https://doi.org/10.4271/2015-01-1538

[32] N. Simmonds, J. Pitman, P. Tsoutsanis, K. Jenkins, A. Caylard, W. Jansen, Complete body aerodynamic study of three vehicles, in: SAE Technical Paper 2017-01-1529, SAE International, 2017. doi:https://doi.org/10.4271/2017-01-1529.

URL https://doi.org/10.4271/2017-01-1529

[33] D. C. Forbes, G. J. Page, M. A. Passmore, A. P. Gaylard, A fully coupled, 6 degree-of-freedom, aerodynamic and vehicle handling crosswind simulation using the drivaer model, SAE Int. J. Passeng. Cars - Mech. Syst. 9 (2) (2016) 710-722. doi:https://doi.org/10.4271/2016-01-1601. URL https://doi.org/10.4271/2016-01-1601

[34] F. R. Menter, M. Kuntz, R. Langtry, Ten years of industrial experience with the sst turbulence model, Turbulence, heat and mass transfer 4 (1) (2003) 625-632.

[35] P. R. Spalart, S. Deck, M. L. Shur, K. D. Squires, M. K. Strelets, A. Travin, A new version of detachededdy simulation, resistant to ambiguous grid densities, Theoretical and computational fluid dynamics 20 (3) (2006) 181.

[36] M. L. Shur, P. R. Spalart, M. K. Strelets, A. K. Travin, A hybrid rans-les approach with delayed-des and wall-modelled les capabilities, International Journal of Heat and Fluid Flow 29 (6) (2008) 1638-1649.

[37] L. Sterken, S. Sebben, L. Löfdahl, Numerical implementation of detached-eddy simulation on a passenger vehicle and some experimental correlation, Journal of Fluids Engineering 138 (9) (2016) 091105.

[38] G. Johl, M. Passmore, P. Render, Design methodology and performance of an indraft wind tunnel, Aeronautical Journal 108 (1087) (2004) 465-473. 
[39] G. Johl, The design and performance if a $1.9 \mathrm{~m} \times 1.3 \mathrm{~m}$ indraft wind tunnel, Ph.D. thesis (2010).

[40] M. Varney, M. Passmore, A. Gaylard, The effect of passive base ventilation on the aerodynamic drag of a generic suv vehicle, SAE International Journal of Passenger Cars - Mechanical Systems 10 (1) (2017) 345-357. doi:https://doi.org/10.4271/2017-01-1548.

URL https://doi.org/10.4271/2017-01-1548

[41] K. Cooper, Closed-test-section wind tunnel blockage corrections for road vehicles. special publication sae sp1176, Society of Automotive Engineers, SAE.

[42] A. Gaylard, A. Kabanovs, J. Jilesen, K. Kirwan, D. Lockerby, Simulation of rear surface contamination for a simple bluff body, Journal of Wind Engineering and Industrial Aerodynamics 165 (2017) 13-22.

[43] M. Grandemange, A. Mary, M. Gohlke, O. Cadot, Effect on drag of the flow orientation at the base separation of a simplified blunt road vehicle, Experiments in fluids 54 (5) (2013) 1529.

[44] E. G. Duell, A. George, Experimental study of a ground vehicle body unsteady near wake, in: SAE Technical Paper 1999-01-0812, SAE International, 1999. doi:10.4271/1999-01-0812.

[45] L. Dalla Longa, O. Evstafyeva, A. Morgans, Bi-modality in the wakes of simplified road vehicles: simulation and feedback control, in: 52nd 3AF International Conference on Applied Aerodynamics, 2017.

[46] A. Islam, A. Gaylard, B. Thornber, A detailed statistical study of unsteady wake dynamics from automotive bluff bodies, Journal of Wind Engineering and Industrial Aerodynamics 171 (2017) 161177. 